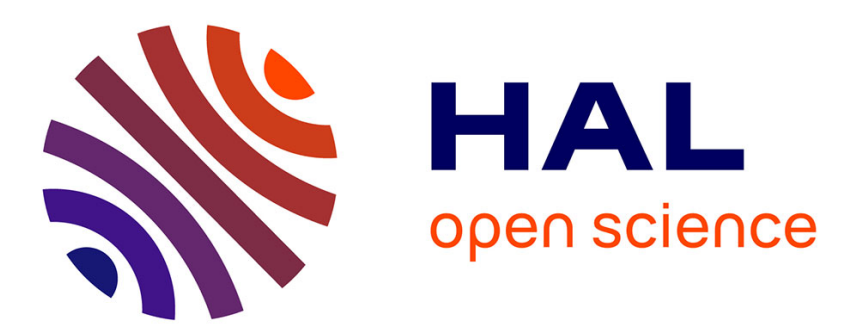

\title{
Large time step and asymptotic preserving numerical schemes for the gas dynamics equations with source terms
}

Christophe Chalons, Samuel Kokh, Mathieu Girardin

\section{To cite this version:}

Christophe Chalons, Samuel Kokh, Mathieu Girardin. Large time step and asymptotic preserving numerical schemes for the gas dynamics equations with source terms. 2012. hal-00718022

\section{HAL Id: hal-00718022 \\ https://hal.science/hal-00718022}

Preprint submitted on 15 Jul 2012

HAL is a multi-disciplinary open access archive for the deposit and dissemination of scientific research documents, whether they are published or not. The documents may come from teaching and research institutions in France or abroad, or from public or private research centers.
L'archive ouverte pluridisciplinaire HAL, est destinée au dépôt et à la diffusion de documents scientifiques de niveau recherche, publiés ou non, émanant des établissements d'enseignement et de recherche français ou étrangers, des laboratoires publics ou privés. 


\title{
Large time step and asymptotic preserving numerical schemes for the gas dynamics equations with source terms
}

\author{
Christophe Chalons ${ }^{* \dagger} \quad$ Mathieu Girardin ${ }^{\ddagger \dagger} \quad$ Samuel Kokh ${ }^{\S \dagger}$
}

February 9, 2012

\begin{abstract}
We propose a large time step and asymptotic preserving scheme for the gas dynamics equations with external forces and friction terms. By asymptotic preserving, we mean that the numerical scheme is able to reproduce at the discrete level the parabolic-type asymptotic behaviour satisfied by the continuous equations. By large time-step, we mean that the scheme is stable under a CFL stability condition driven by the (slow) material waves, and not by the (fast) acoustic waves as it is customary in Godunov-type schemes. Numerical evidences are proposed and show a gain of several orders of magnitude in both accuracy and efficiency.
\end{abstract}

\section{Introduction}

We are interested in the simulation of subsonic compressible flows in a specific regime where the (main) driving phenomena are stiff source terms and material transport. More precisely, we consider the system of gas dynamics with external body forces and friction. Such flow configuration may be encountered in several industrial processes like the flows involved within the core of a nuclear power plant.

We propose here a method that fulfils our task by addressing three issues. First we require our method to enable the use of large time steps in order to avoid classic Courant-Friedrichs-Lewy (CFL) restriction based on the (fast) acoustic waves of the model. Second, we want our method to accurately approximate (slow) waves that account for material transport. Third, the discretization of the stiff source terms with large time steps may severely affect the accuracy of the method. We propose to overcome this difficulty by imposing an even stronger property on our numerical scheme. Indeed, when one considers the asymptotic regime obtained for both long time and large friction coefficients, the solution of the system is formally expected to behave like the solution of a typical parabolic system. We aim at deriving a scheme that preserves this property for the discrete approximate of the solution. Such property is referred to as an asymptotic preserving (AP) property. Since its introduction in the pioneer work of [23, 25, the notion of AP numerical schemes has been investigated and implemented in the past years in a wide range of context stemming from hydrodynamics with radiative transfer [22, 8, 7, 2, 4, multiscale kinetics [24], diffusive limit of the transport equation [26, 29] to problems similar to our model [6, 4, 11. Without being exhaustive, we also refer the reader to [9, 16, 3, 1, 26.

Classic means to fulfil our first requirement for avoiding CFL based on the acoustic waves, consists in deriving an implicit in time discretization. Unfortunately this usually induces more numerical diffusion, including for the approximation of the material waves. In order to meet both first and second need, we propose a mixed implicit-explicit strategy: the terms responsible for the acoustic waves receive a time implicit treatment while the ones responsible for the transport waves are treated by an explicit update. This task is achieved by means of a Lagrange-Projection [19] algorithm as in [14. This approach provides a natural decoupling of the acoustic waves and the material waves. An approximation based on a relaxation strategy [31, 27, 15, 13, 12, 5] provides a simple mean to circumvent the nonlinearities involved with the equation of state of the fluid.

\footnotetext{
*Université Paris Diderot-Paris 7 \& Laboratoire J.-L. Lions, U.M.R. 7598 UMPC, Boîte courrier 187, 75252 Paris Cedex 05, France. E-mail: christophe_chalons@ljll.univ-paris-diderot.fr.

${ }^{\dagger}$ LRC MANON, Laboratoire de Recherche Conventionné CEA/DEN/DANS/DM2S and UPMC-CNRS/LJLL.

‡DEN/DANS/DM2S/STMF/LMEC - CEA Saclay, bât. 454 PC 47, 91191 Gif sur Yvette Cedex, France. Email: mathieu.girardin@cea.fr.

§DEN/DANS/DM2S/STMF/LMEC - CEA Saclay, bât. 454 PC 47, 91191 Gif sur Yvette Cedex, France. Email: samuel.kokh@cea.fr.
} 
The effects of both gravity and friction source terms are then incorporated into the solver thanks to the concept of simple approximate Riemann solver and consistency with the integral form introduced by Gallice [17, [18. This powerful method allows to account for both source terms and convective fluxes at the same time. It has been applied in [11 for deriving an explicit scheme for the same flow model. We shall show that this approach can be followed within a mixed implicit-explicit framework for designing an AP scheme.

The outline of the paper is as follows. In the next section, we give the model under consideration and its parabolic-type asymptotic limit. In section 3 , we first briefly recall the Lagrange-Projection decomposition and the pressure relaxation strategy. We then recall the concepts of simple approximate Riemann solver and consistency in the integral sense in section 4. The last part of this section gives the explicit in time numerical scheme and section 5 focuses on the Lagrangian system. At last, the implicit in time scheme for the Lagrangian system is given in section 6 and the overall mixed implicit-explicit scheme is described in section 7 . Finally, section 8 gives the main properties of the mixed implicit-explicit scheme and the last section provides some numerical illustrations.

\section{Governing equations and asymptotic behaviour}

The gas dynamics equations with gravity and friction terms in Eulerian coordinates are given by

$$
\left\{\begin{array}{l}
\partial_{t} \rho+\partial_{x}(\rho u)=0, \\
\partial_{t}(\rho u)+\partial_{x}\left(\rho u^{2}+p\right)=\rho(g-\alpha u), \\
\partial_{t}(\rho E)+\partial_{x}((\rho E+p) u)=\rho u(g-\alpha u),
\end{array}\right.
$$

where $\rho, u$ and $E$ denote the density, the velocity and the total energy of the fluid, $g$ the gravitational acceleration and $\alpha$ the friction parameter. The pressure law $p=p(\rho, e)$ is assumed to be a given function of the density

$\rho$ and the internal energy $e$ defined by $e=E-\frac{u^{2}}{2}$, satisfying the usual Weyl assumptions 32]. Under these assumptions and when the source terms are omitted, (1) is shown to be strictly hyperbolic over the phase space $\Omega$ given by

$$
\Omega=\left\{(\rho, \rho u, \rho E)^{T} \in \mathbb{R}^{3}, \rho>0, e>0\right\}
$$

with eigenvalues given by

$$
\lambda_{1}=u-c<\lambda_{2}=u<\lambda_{3}=u+c,
$$

where $c=\left[(\partial p / \partial \rho)_{\epsilon}+\left(p / \rho^{2}\right)(\partial p / \partial \epsilon)_{\rho}\right]^{1 / 2}$ is the sound speed. Moreover, the characteristic fields associated with $\lambda_{1}$ and $\lambda_{3}$ are genuinely non linear while the characteristic field associated with $\lambda_{2}$ is linearly degenerate. Let us also recall that $\lambda_{1}$ and $\lambda_{3}$ give rise to the so-called acoustic waves, while $\lambda_{2}$ is associated to the transport phenomenon. We refer for instance the reader to [19] for more details.

Let $s=s(\rho, e)$ be the strictly convex mathematical specific entropy which satisfies

$$
-T d s=d e+p \mathrm{~d}\left(\frac{1}{\rho}\right),
$$

where $T>0$ is the temperature. We obtain for a smooth solution of (1) the following equation

$$
\partial_{t}(\rho s)+\partial_{x}(\rho s u)=0
$$

We are particularly interested in studying the long time behavior of (1) when the friction parameter goes to infinity. Let $\epsilon$ be a small positive parameter. We model this flow regime by replacing $\alpha$ with $\alpha / \epsilon$ with a slight abuse of notation and by performing the change of variable $t^{\prime}=\epsilon t$ in the system (1). We obtain the system

$$
\left\{\begin{aligned}
\epsilon \partial_{t^{\prime}} \rho+\partial_{x}(\rho u) & =0 \\
\epsilon \partial_{t^{\prime}}(\rho u)+\partial_{x}\left(\rho u^{2}+p\right) & =\rho\left(g-\frac{\alpha}{\epsilon} u\right), \\
\epsilon \partial_{t^{\prime}}(\rho E)+\partial_{x}((\rho E+p) u) & =\rho u\left(g-\frac{\alpha}{\epsilon} u\right) .
\end{aligned}\right.
$$

Let us assume that the velocity $u$ admits an asymptotic expansion in powers of $\epsilon$ of the following form

$$
u=u^{0}+\epsilon u^{1}+\mathcal{O}\left(\epsilon^{2}\right) .
$$


Multiplying the second equation by $\epsilon$ and letting $\epsilon$ go to 0 first gives $u^{0}=0$. Then inserting $u=\epsilon u^{1}+\mathcal{O}\left(\epsilon^{2}\right)$ in the first equation, dividing by $\epsilon$ and letting $\epsilon$ go to 0 gives

$$
\partial_{t^{\prime}} \rho+\partial_{x} \rho u^{1}=0 .
$$

If we now insert $u=\epsilon u^{1}+\mathcal{O}\left(\epsilon^{2}\right)$ in the second equation and let $\epsilon$ go to 0 , we get

$$
\partial_{x} p=\rho g-\rho \alpha u^{1} .
$$

At last, inserting $u=\epsilon u^{1}+\mathcal{O}\left(\epsilon^{2}\right)$ in the third equation, dividing by $\epsilon$ and letting $\epsilon$ go to 0 gives

$$
\partial_{t^{\prime}}(\rho e)+\partial_{x}\left(\rho e u^{1}+p u^{1}\right)=\rho u^{1}\left(g-\alpha u^{1}\right) .
$$

The long time behaviour of the solutions of (1) for large friction coefficients is then given by the following system of partial differential equations

$$
\left\{\begin{array}{l}
\partial_{t^{\prime}} \rho+\partial_{x}\left(\rho u^{1}\right)=0 \\
\partial_{x} p=\rho\left(g-\alpha u^{1}\right) \\
\partial_{t^{\prime}}(\rho e)+\partial_{x}\left((\rho e+p) u^{1}\right)=\rho u^{1}\left(g-\alpha u^{1}\right),
\end{array}\right.
$$

where we note that in comparison to (1) the flow speed $u$ has been replaced by its first order corrector $u^{1}$ in both the mass flux of the first equation and the friction term of the second equation. This observation will play a crucial role in the forthcoming developments. In addition, we note that this model has to be understood as the diffusive or parabolic limit of the hyperbolic model (1) since the second derivative of the pressure $p$ naturally appears in the first equation of the limit system using its second equation :

$$
\partial_{t^{\prime}} \rho+\partial_{x}\left(\frac{\rho g-\partial_{x} p}{\alpha}\right)=0
$$

From a numerical point of view and as already said in the previous section, one of our objectives is to preserve this asymptotic behaviour at the discrete level. In other words, we aim at proposing a consistent numerical scheme for (1) leading to a consistent numerical scheme for (4) when $\epsilon$ goes to zero and up to the expected changes of variables. Before proceeding and to conclude this section, let us formally rephrase this property in terms of limits with respect to the small parameter $\epsilon$ and to the time and space steps $\Delta t, \Delta x$ used in the numerical approximation. Let us denote $M^{\epsilon}$ the initial model (1), $M^{0}$ the limit model (4), $S_{\Delta t, \Delta x}^{\epsilon}$ a consistent numerical scheme for 1 and $S_{\Delta t, \Delta x}^{0}$ its asymptotic limit. Recall that consistency of $S_{\Delta t, \Delta x}^{\epsilon}$ means that

$$
\lim _{\Delta t, \Delta x \rightarrow 0} S_{\Delta t, \Delta x}^{\epsilon}=M^{\epsilon},
$$

for all $\epsilon>0$. By definition and with a little abuse in the notations, $S_{\Delta t, \Delta x}^{\epsilon}$ is said to be asymptotic preserving if $S_{\Delta t, \Delta x}^{0}$ is consistent with $M^{0}$, that is

$$
\lim _{\Delta t, \Delta x \rightarrow 0} S_{\Delta t, \Delta x}^{0}=M^{0}
$$

or equivalently

$$
\lim _{\Delta t, \Delta x \rightarrow 0} \lim _{\epsilon \rightarrow 0} S_{\Delta t, \Delta x}^{\epsilon}=\lim _{\epsilon \rightarrow 0} M^{\epsilon} .
$$

In other words, the asymptotic preserving property is formally equivalent to the following order of limits interchange property :

$$
\lim _{\Delta t, \Delta x \rightarrow 0} \lim _{\epsilon \rightarrow 0} S_{\Delta t, \Delta x}^{\epsilon}=\lim _{\epsilon \rightarrow 0} \lim _{\Delta t, \Delta x \rightarrow 0} S_{\Delta t, \Delta x}^{\epsilon} .
$$

From a practical point of view this equality formally means that for large friction coefficients, or equivalently for small values of $\epsilon$, an asymptotic preserving scheme is expected to give good numerical results even for reasonable mesh sizes (with respect to $\epsilon$ ). This will be observed in the last section devoted to the numerical experiments.

\section{Lagrange-Projection approach and relaxation procedure}

In this section we briefly recall the so-called Lagrange-Projection strategy applied to (1) and propose a relaxation procedure for approximating the solutions of the underlying Lagrangian system. As motivated in the introduction, these are two key ingredients of the method we propose, together with the notion of consistency in the integral sense that will be recalled in the next section. Let us begin with the Lagrange-Projection decomposition. 


\subsection{Lagrange-Projection decomposition}

We describe here a procedure that allows to approximate the evolution of the system (1) over a time interval $\left[t_{0}, t_{0}+\Delta t\right]$. The guideline of the method consists in decoupling the terms responsible for the acoustic waves and the transport waves. By using the chain rule for the space derivatives we split up the operators of system (1) and obtain two subsystems. The first subsystem describes the transport process and reads

$$
\left\{\begin{array}{l}
\partial_{t} \rho+u \partial_{x} \rho=0 \\
\partial_{t}(\rho u)+u \partial_{x}(\rho u)=0 \\
\partial_{t}(\rho E)+u \partial_{x}(\rho E)=0
\end{array}\right.
$$

The second subsystem accounts for acoustic, gravity and friction effects, namely

$$
\left\{\begin{array}{l}
\partial_{t} \rho+\rho \partial_{x} u=0 \\
\partial_{t}(\rho u)+\rho u \partial_{x} u+\partial_{x} p=\rho(g-\alpha u) \\
\partial_{t}(\rho E)+\rho E \partial_{x} u+\partial_{x}(p u)=\rho u(g-\alpha u) .
\end{array}\right.
$$

If we note $\tau=\frac{1}{\rho}$ the specific volume, the above system also reads

$$
\left\{\begin{aligned}
\partial_{t} \tau-\tau \partial_{x} u & =0 \\
\partial_{t} u+\tau \partial_{x} p & =g-\alpha u \\
\partial_{t} E+\tau \partial_{x}(p u) & =u(g-\alpha u) .
\end{aligned}\right.
$$

Then for $t \in\left[t_{0}, t_{0}+\Delta t\right]$, we propose to approximate $\tau(x, t) \partial_{x}$. by $\tau\left(x, t_{0}\right) \partial_{x}$. in (6). If one introduces the mass variable $m$ defined by $\mathrm{d} m=\tau\left(x, t_{0}\right)^{-1} \mathrm{~d} x$, we obtain

$$
\left\{\begin{array}{l}
\partial_{t} \tau-\partial_{m} u=0 \\
\partial_{t} u+\partial_{m} p=g-\alpha u \\
\partial_{t} E+\partial_{m}(p u)=u(g-\alpha u)
\end{array}\right.
$$

that will be referred to as the Lagrangian system. Let us note that system (7) is consistent the usual form of the gas dynamics equations in Lagrangian coordinates with friction and gravity terms. We refer for instance the reader to [19] for more details. It is worth noticing that (7) is easily shown to be hyperbolic over the phase space $\Omega^{\mathrm{Lag}}$ given by

$$
\Omega^{\mathrm{Lag}}=\left\{(\tau, u, E)^{T} \in \mathbb{R}^{3}, \tau>0, e>0\right\},
$$

with eigenvalues given by

$$
\lambda_{1}^{\mathrm{Lag}}=-\rho c<\lambda_{2}^{\mathrm{Lag}}=0<\lambda_{3}^{\mathrm{Lag}}=\rho c,
$$

where $c$ still denotes the sound speed. Here again, the extreme characteristic fields associated with $\lambda_{1}^{\mathrm{Lag}}$ and $\lambda_{3}^{\mathrm{Lag}}$ are genuinely non linear while the intermediate characteristic field associated with $\lambda_{2}^{\mathrm{Lag}}$ is linearly degenerate. Importantly, we note that the sound speed only appears in the characteristic speeds of this Lagrangian model. The flow speed $u$ is no longer present but is on the other hand the unique characteristic velocity of the first subsystem (5). System (7) (respectively (5)) then clearly encompasses to the so-called acoustic (resp. material or transport) waves. From a numerical point of view, the scheme associated with this decomposition simply consists of an usual two-step splitting strategy where (7) is solve in the first step and (5) in the second one. Recall that the idea will be here to propose a time implicit treatment of the Lagrangian system (7) to avoid a too restrictive CFL condition involving the sound speed $c$ and an explicit treatment of the transport part (5) to keep accuracy on the material waves. A key ingredient to get a low cost implicit scheme for the Lagrangian system will rely on a relevant pressure relaxation approximation described in the next section.

\subsection{Relaxation approximation}

We propose in this section a relaxation approximation of the Lagragian system (7). The main objective is to overcome the non linearities that make difficult the resolution of this system. From a numerical point of view, this strategy will be used to design a low cost time implicit treatment. We first consider the model neglecting the source terms and then extend the approach to the full model. 


\subsubsection{Relaxation approximation of the homogeneous model}

The design principle of the so-called pressure relaxation methods is to introduce a larger system than the original one but easier to solve. More precisely, the objective is to discard the non linearities induced by the pressure law $p=p(\rho, e)$. Such a strategy is now well known in the literature and we refer for instance the reader to 31, 27, 15, 13, 12, 5 and the references therein. To do so, we introduce a new variable $\Pi$ that can be seen as a linearization of the pressure $p$ and that is considered as a new unknown. In particular, it evolves according to its own partial differential equation. More precisely, we propose the following relaxation system for (7) when the source terms are omitted :

$$
\left\{\begin{array}{l}
\partial_{t} \tau-\partial_{m} u=0, \\
\partial_{t} u+\partial_{m} \Pi=0, \\
\partial_{t} \Pi+a^{2} \partial_{m} u=\lambda(p-\Pi), \\
\partial_{t} E+\partial_{m}(\Pi u)=0,
\end{array}\right.
$$

where $a$ is a constant to be precised and $\lambda$ the relaxation parameter. At least formally, we observe that in the asymptotic regime $\lambda \rightarrow+\infty$ we have $\Pi \rightarrow p$ and we recover the initial system 77 without the source terms. In order to prevent this relaxation procedure form instabilities, it is now well established that $a$ must be chosen sufficiently large and according to the subcharacteristic condition

$$
a>\max (\rho c),
$$

for all the states under consideration (see for instance [12] for a rigorous proof). In addition, it can be easily proved that (8) with $\lambda=0$ is strictly hyperbolic with three eigenvalues given by $-a, 0$ and $a$ which are nothing but approximations of the exact eigenvalues $-\rho c, 0$ and $\rho c$ for system (7). Then and in particular, the subcharacteristic condition means that information propagates faster in the relaxation model. More importantly, the characteristic fields associated with these new eigenvalues are shown to be linearly degenerate. This property allows to solve analytically the Riemann problem associated with (8) when $\lambda=0$, that is when considering an initial data made of two constant states separated by an initial discontinuity. This property justifies by itself the introduction of the proposed relaxation model and its simplicity. If we go further into the details and as it is customary, the exact Riemann solutions are self-similar and made of three contact discontinuities propagating with velocities $-a, a$ and 0 and separating two intermediates states.

From a numerical point of view, the numerical strategy for approximating the solutions of (7) using (8) consists in first solving (8) with $\lambda=0$, that is

$$
\left\{\begin{array}{l}
\partial_{t} \tau-\partial_{m} u=0, \\
\partial_{t} u+\partial_{m} \Pi=0, \\
\partial_{t} \Pi+a^{2} \partial_{m} u=0, \\
\partial_{t} E+\partial_{m}(\Pi u)=0,
\end{array}\right.
$$

and then to take into account the source term

$$
\left\{\begin{array}{l}
\partial_{t} \tau=0, \\
\partial_{t} u=0, \\
\partial_{t} \Pi=\lambda(p-\Pi), \\
\partial_{t} E=0,
\end{array}\right.
$$

in the asymptotic regime $\lambda \rightarrow+\infty$. Which amounts to set

$$
\Pi=p(\rho, E),
$$

before solving again 10 as the time goes on. The new variable $\Pi$ is said to be at equilibrium.

To conclude this section, notice that the self-similar Riemann solutions associated with (10) being explicitly known, it is natural to use an exact Godunov scheme to numerically solve 10 . See for instance the references above for more details.

\subsubsection{Relaxation approximation of the Lagrangian system with source terms}

Let $a$ be a real parameter chosen in agreement with the subcharacteristic condition (9). In order to approximate the solution of (7), we propose to supplement (10) with the friction and gravity terms so that we now have to 
solve

$$
\left\{\begin{array}{l}
\partial_{t} \tau-\partial_{m} u=0 \\
\partial_{t} u+\partial_{m} \Pi=g-\alpha u \\
\partial_{t} \Pi+a^{2} \partial_{m} u=0 \\
\partial_{t} E+\partial_{m}(\Pi u)=u(g-\alpha u) .
\end{array}\right.
$$

Let us underline that the solutions of the Riemann problem associated with (11) are neither self-similar nor explicitly known anymore. This makes the use of the exact Godunov method quite a complex task. In what follows, we decide nevertheless to approximate the non self-similar Riemann solutions to (11) by self-similar approximate Riemann solutions and to use an approximate Godunov-type method for solving (11). In order to guarantee the consistency of the proposed self-similar approximate Riemann solutions to (11) with the exact ones, we will impose a generalized notion of consistency in the integral sense due to Gallice [17, 18] and adapted to systems with source terms. This is recalled in the next section.

To conclude this section, let us observe that (11) can be given the following equivalent form

$$
\left\{\begin{array}{l}
\partial_{t} \tau-\partial_{m} u=0 \\
\partial_{t} \vec{w}+a \partial_{m} \vec{w}=a(g-\alpha u) \\
\partial_{t} \overleftarrow{w}-a \partial_{m} \overleftarrow{w}=-a(g-\alpha u) \\
\partial_{t} E+\partial_{m}(\Pi u)=u(g-\alpha u)
\end{array}\right.
$$

where the new variables $\vec{w}$ and $\overleftarrow{w}$ are defined by

$$
\vec{w}=\Pi+a u, \quad \overleftarrow{w}=\Pi-a u
$$

These quantities are nothing but the strong Riemann invariants associated with the characteristic speeds $\pm a$ of the relaxation system (11) when the source terms are omitted. The closure relations for (12) are naturally given by

$$
u=\frac{\vec{w}-\overleftarrow{w}}{2 a}, \quad \Pi=\frac{\vec{w}+\overleftarrow{w}}{2}
$$

This new formulation will be used hereafter to define the proposed implicit in time numerical strategy.

\section{Consistency in the integral sense and explicit in time Godunov- type scheme}

We briefly recall in this section the notion of consistency in the integral sense of a self-similar approximate Riemann solver for a given set of hyperbolic equations with source terms that we write in the following condensed form

$$
\partial_{t} \mathbf{U}+\partial_{x} \mathbf{F}(\mathbf{U})=\mathbf{S}(\mathbf{U})
$$

supplemented with the validity of an entropy inequality

$$
\partial_{t} \eta+\partial_{x} q \leq 0
$$

where $(\eta, q)$ is a strictly convex entropy-entropy flux pair. We also derive the corresponding explicit in time Godunov-type scheme for approximating the solutions to (15) and refer to [17, 18, for the details.

Solving the Riemann problem amounts to find the solution to 15 with the following piecewise constant initial data

$$
\mathbf{U}(x, t=0)=\left\{\begin{array}{lll}
\mathbf{U}_{L} & \text { if } \quad x<0, \\
\mathbf{U}_{R} & \text { if } \quad x>0,
\end{array}\right.
$$

for any given $\mathbf{U}_{L}$ and $\mathbf{U}_{R}$ in the phase space. Unlike the homogeneous case corresponding to the choice $\mathbf{S}(\mathbf{U})=0$, the exact Riemann solution that we denote $\mathbf{U}\left(x, t ; \mathbf{U}_{L}, \mathbf{U}_{R}\right)$ is not self-similar. Notice however that an approximate Riemann solver $\mathbf{W}\left(\frac{x}{t} ; \mathbf{U}_{L}, \mathbf{U}_{R}\right)$ may be self-similar as in the homogeneous case provided that some consistency relations are imposed. More precisely, let us consider a simple approximate Riemann solver 


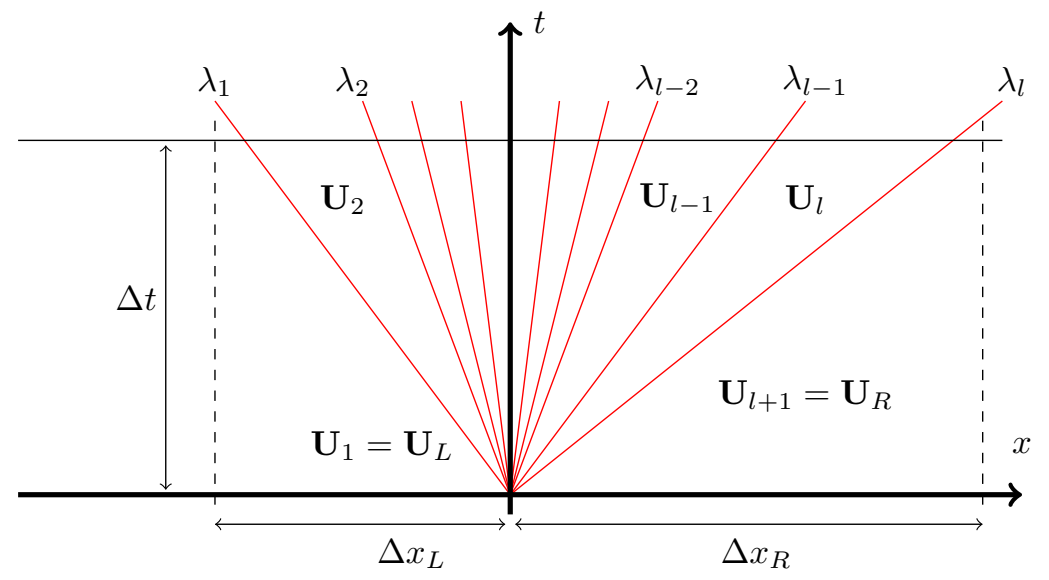

Figure 1: Wave structure of the approximate Riemann solver in the $(x, t)$-plane.

$\mathbf{W}\left(\frac{x}{t} ; \mathbf{U}_{L}, \mathbf{U}_{R}\right)$ made of $l+1$ intermediate states $\mathbf{U}_{k}$ separated by discontinuities propagating with velocities $\lambda_{k}$ (see figure 1), namely

$$
\mathbf{W}\left(\frac{x}{t} ; \mathbf{U}_{L}, \mathbf{U}_{R}\right)= \begin{cases}\mathbf{U}_{1}=\mathbf{U}_{L}, & \frac{x}{t}<\lambda_{1}, \\ \vdots & \lambda_{k-1}<\frac{x}{t}<\lambda_{k}, \\ \mathbf{U}_{k}, & \\ \vdots & \frac{x}{t}>\lambda_{l} .\end{cases}
$$

From Gallice [17, 18, if $\Delta x=\frac{1}{2}\left(\Delta x_{L}+\Delta x_{R}\right)$ with $\Delta x_{L}>0, \Delta x_{R}>0$ and $\Delta t>0$ are respectively space and time steps that verify the CFL condition

$$
\max _{1 \leq k \leq l}\left|\lambda_{k}\right| \frac{\Delta t}{\min \left(\Delta x_{L}, \Delta x_{R}\right)} \leq \frac{1}{2}
$$

the approximate Riemann solver is said to be consistent with the integral form of (15) over the interval $\left[\frac{-\Delta x_{L}}{2}, \frac{\Delta x_{R}}{2}\right]$ if the integral of 117 approximates correctly the integral of the exact solution in the sense that there exists a function $\widetilde{\mathbf{S}}$ such that

$$
\mathbf{F}\left(\mathbf{U}_{R}\right)-\mathbf{F}\left(\mathbf{U}_{L}\right)-\Delta x \widetilde{\mathbf{S}}\left(\Delta x, \Delta t ; \mathbf{U}_{L}, \mathbf{U}_{R}\right)=\sum_{k=1}^{l} \lambda_{k}\left(\mathbf{U}_{k+1}-\mathbf{U}_{k}\right),
$$

where $\widetilde{\mathbf{S}}\left(\Delta x, \Delta t ; \mathbf{U}_{L}, \mathbf{U}_{R}\right)$ is consistent with the source terms $\mathbf{S}(\mathbf{U})$ in the sense that

$$
\lim _{\substack{\mathbf{U}_{L}, \mathbf{U}_{R} \rightarrow \mathbf{U} \\ \Delta t, \Delta x \rightarrow 0}} \widetilde{\mathbf{S}}\left(\Delta x, \Delta t ; \mathbf{U}_{L}, \mathbf{U}_{R}\right)=\mathbf{S}(\mathbf{U})
$$

Hereafter and using very classical notations, $\left(\Delta x_{j}\right)_{j \in \mathbb{Z}}$ and $\Delta t$ represent the constant time and variable space steps of the mesh under consideration for defining the approximate solutions. More precisely and in order to define the Godunov-type scheme associated with this approximate Riemann solver, we define the mesh interfaces $x_{j+1 / 2}=x_{j-1 / 2}+\Delta x_{j}$ for $j \in \mathbb{Z}$, and the intermediate times $t^{n}=n \Delta t$ for $n \in \mathbb{N}$. Note that $\Delta x$ in 19 then plays the role of $\Delta x_{j+1 / 2}=\frac{1}{2}\left(\Delta x_{j}+\Delta x_{j+1}\right)$. In the sequel, $\mathbf{U}_{j}^{n}$ denotes the approximate value of $\mathbf{U}$ at time $t^{n}$ and on the cell $\left[x_{j-1 / 2}, x_{j+1 / 2}\right)$. For $n=0$ and $j \in \mathbb{Z}$, we set $\mathbf{U}_{j}^{0}=\frac{1}{\Delta x} \int_{x_{j-1 / 2}}^{x_{j+1 / 2}} \mathbf{U}_{0}(x) \mathrm{d} x$ where $\mathbf{U}_{0}(x)$ is the 
initial condition. Then, the explicit in time Godunov-type scheme reads

$$
\left\{\begin{array}{l}
\mathbf{U}_{j}^{n+1}=\mathbf{U}_{j}^{n}-\frac{\Delta t}{\Delta x_{j}}\left(\mathbf{F}_{j+\frac{1}{2}}^{n}-\mathbf{F}_{j-\frac{1}{2}}^{n}\right)+\frac{\Delta t}{2}\left(\frac{\Delta x_{j+1 / 2}}{\Delta x_{j}} \mathbf{S}_{j+\frac{1}{2}}^{n}+\frac{\Delta x_{j-1 / 2}}{\Delta x_{j}} \mathbf{S}_{j-\frac{1}{2}}^{n}\right), \\
\mathbf{F}_{j+\frac{1}{2}}^{n}=\mathbf{F}\left(\mathbf{U}_{j}^{n}, \mathbf{U}_{j+1}^{n}\right), \\
\mathbf{S}_{j+\frac{1}{2}}^{n}=\widetilde{\mathbf{S}}\left(\Delta x_{j+1 / 2}, \Delta t ; \mathbf{U}_{j}^{n}, \mathbf{U}_{j+1}^{n}\right),
\end{array}\right.
$$

with

$$
\mathbf{F}\left(\mathbf{U}_{L}, \mathbf{U}_{R}\right)=\frac{1}{2}\left\{\mathbf{F}\left(\mathbf{U}_{L}\right)+\mathbf{F}\left(\mathbf{U}_{R}\right)-\sum_{k=1}^{l}\left|\lambda_{k}\right|\left(\mathbf{U}_{k+1}-\mathbf{U}_{k}\right)\right\} .
$$

As far as the consistency with the entropy inequality $(16)$ is concerned, the simple approximate Riemann solver is said to be consistent with the integral form of 116 if and only if there exists a function $\widetilde{\sigma}$ such that under the CFL condition 18 we have

$$
q\left(\mathbf{U}_{R}\right)-q\left(\mathbf{U}_{L}\right)-\Delta x \widetilde{\sigma}\left(\Delta x, \Delta t ; \mathbf{U}_{L}, \mathbf{U}_{R}\right) \leq \sum_{k=1}^{l} \lambda_{k}\left(\eta\left(\mathbf{U}_{k+1}\right)-\eta\left(\mathbf{U}_{k}\right)\right),
$$

with

$$
\lim _{\substack{\mathbf{U}_{L}, \mathbf{U}_{R} \rightarrow \mathbf{U} \\ \Delta t, \Delta x \rightarrow 0}} \tilde{\sigma}\left(\Delta x, \Delta t ; \mathbf{U}_{L}, \mathbf{U}_{R}\right)=0 .
$$

Then, the numerical scheme defined by (20) satisfies the following discrete entropy inequality

$$
\left\{\begin{array}{l}
\eta\left(\mathbf{U}_{j}^{n+1}\right) \leq \eta\left(\mathbf{U}_{j}^{n}\right)-\frac{\Delta t}{\Delta x_{j}}\left(q_{j+\frac{1}{2}}^{n}-q_{j-\frac{1}{2}}^{n}\right)+\frac{\Delta t}{2}\left(\frac{\Delta x_{j-1 / 2}}{\Delta x_{j}} \sigma_{j-\frac{1}{2}}^{n}+\frac{\Delta x_{j+1 / 2}}{\Delta x_{j}} \sigma_{j+\frac{1}{2}}^{n}\right), \\
q_{j+\frac{1}{2}}^{n}=\widetilde{q}\left(\mathbf{U}_{j}^{n}, \mathbf{U}_{j+1}^{n}\right) \\
\sigma_{j+\frac{1}{2}}^{n}=\widetilde{\sigma}\left(\Delta x_{j+1 / 2}, \Delta t ; \mathbf{U}_{j}^{n}, \mathbf{U}_{j+1}^{n}\right)
\end{array}\right.
$$

with

$$
\widetilde{q}\left(\mathbf{U}_{L}, \mathbf{U}_{R}\right)=\frac{1}{2}\left\{q\left(\mathbf{U}_{L}\right)+q\left(\mathbf{U}_{R}\right)-\sum_{k=1}^{l}\left|\lambda_{k}\right|\left(S\left(\mathbf{U}_{k+1}\right)-S\left(\mathbf{U}_{k}\right)\right)\right\} .
$$

The CFL condition associated with this explicit in time Godunov-type scheme naturally reads

$$
\max _{1 \leq k \leq l}\left|\lambda_{k}\left(\mathbf{U}_{j}^{n}, \mathbf{U}_{j+1}^{n}\right)\right| \frac{\Delta t}{\min \left(\Delta x_{j}, \Delta x_{j+1}\right)} \leq \frac{1}{2},
$$

for all $j$. Again, we refer to [17, 18, 11] for more details.

\section{Application to the Lagrangian system and explicit in time Godunov- type scheme}

We suppose again that $a$ is a parameter that complies with the subcharacteristic constraint (9). We consider a step $\Delta m$ of the space variable expressed through the mass variable and a time step $\Delta t$. The objective of this section is to define a consistent simple approximate Riemann solver for 12. We have in this case

$$
\mathbf{U}=\left(\begin{array}{c}
\tau \\
\vec{w} \\
\overleftarrow{w} \\
E
\end{array}\right), \quad \mathbf{F}(\mathbf{U})=\left(\begin{array}{c}
-u \\
a \vec{w} \\
-a \overleftarrow{w} \\
\Pi u
\end{array}\right), \quad \mathbf{S}(\mathbf{U})=\left(\begin{array}{c}
0 \\
a(g-\alpha u) \\
-a(g-\alpha u) \\
u(g-\alpha u)
\end{array}\right), \quad \mathbf{F}\left(\mathbf{U}_{L}, \mathbf{U}_{R}\right)=\left(\begin{array}{c}
F^{\tau}\left(\mathbf{U}_{L}, \mathbf{U}_{R}\right) \\
F^{\vec{w}}\left(\mathbf{U}_{L}, \mathbf{U}_{R}\right) \\
F^{\overleftarrow{w}}\left(\mathbf{U}_{L}, \mathbf{U}_{R}\right) \\
F^{E}\left(\mathbf{U}_{L}, \mathbf{U}_{R}\right)
\end{array}\right)
$$




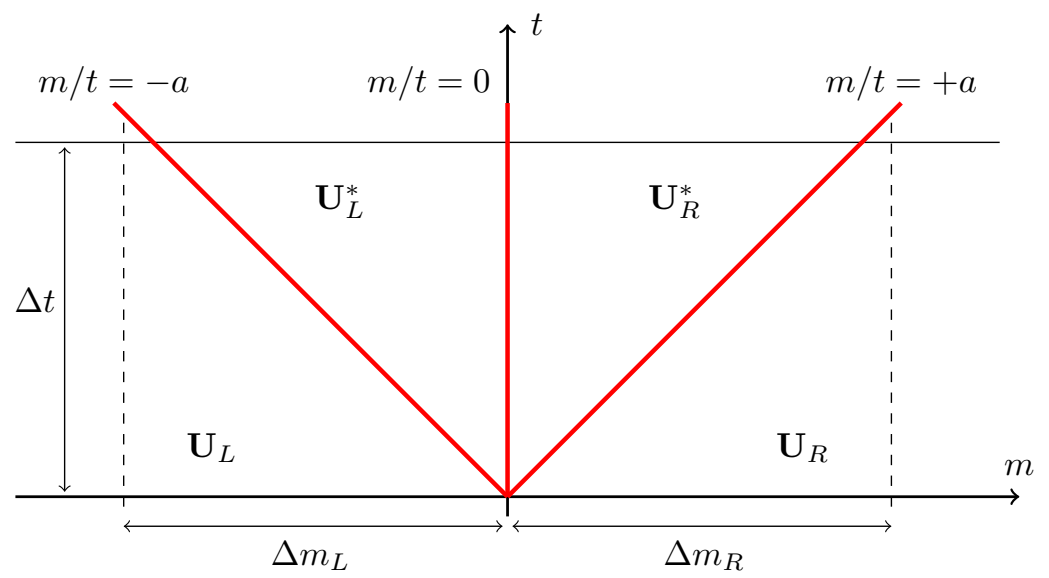

Figure 2: Wave structure of the approximate Riemann solver for the Lagrangian system (12) in the $(m, t)$-plane.

Note in particular that introducing the notation $\mathbf{F}(\mathbf{U})=\left(F^{\tau}, F^{\vec{w}}, F^{\overleftarrow{w}}, F^{E}\right)^{T}$, the energy flux satisfies the relation

$$
F^{E}=-\frac{F^{\vec{w}}+F^{\overleftarrow{w}}}{2 a} F^{\tau}
$$

This relation will be used in the calculations below.

In order to mimic the self-similar solution to 12 when the source terms are omitted, we propose to consider a simple approximate Riemann solver made of 3 waves (see figure 2), namely a stationary wave and two waves propagating with velocities $\pm a$ :

$$
\mathbf{W}\left(\frac{m}{t} ; \mathbf{U}_{L}, \mathbf{U}_{R}\right)= \begin{cases}\mathbf{U}_{L}, & \frac{m}{t}<-a, \\ \mathbf{U}_{L}^{*}, & -a<\frac{m}{t}<0, \\ \mathbf{U}_{R}^{*}, & 0<\frac{m}{t}<a, \\ \mathbf{U}_{R}, & \frac{m}{t}>a .\end{cases}
$$

Following [11, we define $\tilde{\mathbf{S}}$ as follows :

$$
\tilde{\mathbf{S}}\left(\Delta m, \Delta t ; \mathbf{U}_{L}, \mathbf{U}_{R}\right)=\left(\begin{array}{c}
0 \\
a(g-\alpha \tilde{u}) \\
-a(g-\alpha \tilde{u}) \\
\tilde{u}(g-\alpha \tilde{u})
\end{array}\right),
$$

where $\tilde{u}$ represents a consistent approximation of the velocity $u$, in the sense that

$$
\lim _{\substack{\mathbf{U}_{L}, \mathbf{U}_{R \rightarrow \mathbf{U}} \\ \Delta t, \Delta x \rightarrow 0}} \tilde{u}\left(\Delta m, \Delta t ; \mathbf{U}_{L}, \mathbf{U}_{R}\right)=u
$$

The definition of $\tilde{u}$ will be specified later on.

We now turn to the definition the intermediate states $\mathbf{U}_{L}^{*}$ and $\mathbf{U}_{R}^{*}$. Each state containing four components, eight relations are expected. As motivated in the previous section, we first impose the consistency relations 19 which gives here

$$
\left\{\begin{aligned}
\left(u_{L}-u_{R}\right) & =-a\left(\tau_{L}^{*}-\tau_{L}\right)+a\left(\tau_{R}-\tau_{R}^{*}\right) \\
a\left(\vec{w}_{R}-\vec{w}_{L}\right)-\Delta m a(g-\alpha \tilde{u}) & =-a\left(\vec{w}_{L}^{*}-\vec{w}_{L}\right)+a\left(\vec{w}_{R}-\vec{w}_{R}^{*}\right) \\
-a\left(\overleftarrow{w}_{R}-\overleftarrow{w}_{L}\right)+\Delta m a(g-\alpha \tilde{u}) & =-a\left(\overleftarrow{w}_{L}^{*}-\overleftarrow{w}_{L}\right)+a\left(\overleftarrow{w}_{R}-\overleftarrow{w}_{R}^{*}\right) \\
\left(\Pi_{R} u_{R}-\Pi_{L} u_{L}\right)-\Delta m \tilde{u}(g-\alpha \tilde{u}) & =-a\left(E_{L}^{*}-E_{L}\right)+a\left(E_{R}-E_{R}^{*}\right)
\end{aligned}\right.
$$

Then, we make the natural choice of imposing the Rankine Hugoniot relations associated with the mass conservation across each wave of the approximate Riemann solver. We get

$$
\left\{\begin{array}{l}
u_{L}-a \tau_{L}=u_{L}^{*}-a \tau_{L}^{*} \\
u_{R}+a \tau_{R}=u_{R}^{*}+a \tau_{R}^{*} \\
u_{L}^{*}=u_{R}^{*}
\end{array}\right.
$$


Note however that (27) provides only two independent relations since the first equation of (26) is a linear combination of the three equations in (27). Then, two equations are still missing. In the sequel we shall note $u^{*}=u_{L}^{*}=u_{R}^{*}$.

In order to account for the source terms, we propose to impose a generalized jump condition across the stationnary wave and associated with the momentum equation in 11. This amounts to take into account the source term at the interface of the initial condition. More precisely, we impose

$$
\Pi_{R}^{*}-\Pi_{L}^{*}=\Delta m(g-\alpha \tilde{u}) .
$$

So that only one equation is now missing. At last and in regards to the energy equation, we then propose to mimic the relation (25) at the discrete level by imposing the following relation on the numerical flux of the Godunov-type method:

$$
F^{E}\left(\mathbf{U}_{L}, \mathbf{U}_{R}\right)=-F^{\tau}\left(\mathbf{U}_{L}, \mathbf{U}_{R}\right) \times \frac{F^{\vec{w}}\left(\mathbf{U}_{L}, \mathbf{U}_{R}\right)+F^{\overleftarrow{w}}\left(\mathbf{U}_{L}, \mathbf{U}_{R}\right)}{2 a}
$$

It remains to define $\tilde{u}$ and following [11] we set

$$
\tilde{u}=u^{*}
$$

This choice aims at proposing the same approximation of the velocity $u$ in the mass flux at the interface (that is $u^{*}$ ) as in the friction term (that is $\tilde{u}$ ). As mentioned in section 2, this is also true at the continuous level for the parabolic system (4). It turns out to be essential in order to obtain the asymptotic preserving property.

At this point, we can now define the intermediate states $\mathbf{U}_{L}^{*}$ and $\mathbf{U}_{R}^{*}$. We have after easy calculations

$$
\left\{\begin{aligned}
u^{*} & =\frac{1}{2 a+\alpha \Delta m}\left(a\left(u_{R}+u_{L}\right)-\left(\Pi_{R}-\Pi_{L}\right)+g \Delta m\right), \\
\tau_{L}^{*} & =\tau_{L}+\frac{u^{*}-u_{L}}{a}, \\
\tau_{R}^{*} & =\tau_{R}+\frac{u_{R}-u^{*}}{a}, \\
\Pi_{R}^{*} & =\frac{\Pi_{R}+\Pi_{L}}{2}-a \frac{u_{R}-u_{L}}{2}+\frac{\left(g-\alpha u^{*}\right) \Delta m}{2}, \\
\Pi_{L}^{*} & =\Pi_{R}^{*}-\left(g-\alpha u^{*}\right) \Delta m, \\
E_{L}^{*} & =E_{L}+\frac{1}{a}\left(p_{L} u_{L}-u^{*}\left(p^{*}-\frac{\Delta m}{2}\left(g-\alpha u^{*}\right)\right)\right), \\
E_{R}^{*} & =E_{R}-\frac{1}{a}\left(p_{R} u_{R}-u^{*}\left(p^{*}+\frac{\Delta m}{2}\left(g-\alpha u^{*}\right)\right)\right),
\end{aligned}\right.
$$

where we have set

$$
p^{*}=\frac{\Pi_{R}+\Pi_{L}}{2}-a \frac{u_{R}-u_{L}}{2}=\frac{\vec{w}_{L}+\overleftarrow{w}_{R}}{2}
$$

Regarding the numerical flux of the Godunov-type scheme, we find

$$
\left\{\begin{array}{l}
F^{\tau}\left(\mathbf{U}_{L}, \mathbf{U}_{R}\right)=-u^{*} \\
F^{\vec{w}}\left(\mathbf{U}_{L}, \mathbf{U}_{R}\right)=a \vec{w}_{L}+\frac{a\left(g-\alpha u^{*}\right) \Delta m}{2}=a^{2} u^{*}+a p^{*} \\
F^{\overleftarrow{w}}\left(\mathbf{U}_{L}, \mathbf{U}_{R}\right)=-a \overleftarrow{w}_{R}+\frac{a\left(g-\alpha u^{*}\right) \Delta m}{2}=a^{2} u^{*}-a p^{*} \\
F^{E}\left(\mathbf{U}_{L}, \mathbf{U}_{R}\right)=p^{*} u^{*}
\end{array}\right.
$$

We now use the above flux definition to derive an explicit in time Godunov-type scheme for 12 . We consider the approximate variable $\mathbf{U}_{j}^{n}$ known for $j \in \mathbb{Z}$ and we set

$$
\Delta m_{j}=\rho_{j}^{n} \Delta x, \quad \Delta m_{j+1 / 2}=\frac{\Delta m_{j}+\Delta m_{j+1}}{2} .
$$

The superscript ${ }^{\text {Lag }}$ will denote the updated values after the approximation of $(12)$. Following $(20)$, we are led 
to the following scheme

$$
\left\{\begin{aligned}
\tau_{j}^{\mathrm{Lag}} & =\tau_{j}^{n}+\frac{\Delta t}{\Delta m_{j}}\left(u_{j+\frac{1}{2}}^{*}-u_{j-\frac{1}{2}}^{*}\right), \\
\vec{w}_{j}^{\mathrm{Lag}} & =\vec{w}_{j}^{n}-a \frac{\Delta t}{\Delta m_{j}}\left(\vec{w}_{j}^{n}-\vec{w}_{j-1}^{n}\right)+\Delta t a \frac{\Delta m_{j-1 / 2}}{\Delta m_{j}}\left(g-\alpha u_{j-\frac{1}{2}}^{*}\right) \\
\overleftarrow{w}_{j}^{\mathrm{Lag}} & =\overleftarrow{w}_{j}^{n}+a \frac{\Delta t}{\Delta m_{j}}\left(\overleftarrow{w}_{j+1}^{n}-\overleftarrow{w}_{j}^{n}\right)-\Delta t a \frac{\Delta m_{j+1 / 2}}{\Delta m_{j}}\left(g-\alpha u_{j+\frac{1}{2}}^{*}\right), \\
E_{j}^{\mathrm{Lag}} & =E_{j}^{n}-\frac{\Delta t}{\Delta m_{j}}\left((u p)_{j+\frac{1}{2}}^{*}-(u p)_{j-\frac{1}{2}}^{*}\right)+g \frac{\Delta t}{\Delta m_{j}} \frac{\Delta m_{j+1 / 2} u_{j+\frac{1}{2}}^{*}+\Delta m_{j-1 / 2} u_{j-\frac{1}{2}}^{*}}{2} \\
& -\alpha \frac{\Delta t}{\Delta m_{j}} \frac{\Delta m_{j+1 / 2}\left(u_{j+\frac{1}{2}}^{*}\right)^{2}+\Delta m_{j-1 / 2}\left(u_{j-\frac{1}{2}}^{*}\right)^{2}}{2}
\end{aligned}\right.
$$

where

$$
u_{j+\frac{1}{2}}^{*}=\frac{1}{2 a+\alpha \Delta m_{j+1 / 2}}\left(\vec{w}_{j}^{n}-\overleftarrow{w}_{j+1}^{n}+g \Delta m_{j+1 / 2}\right), \quad p_{j+\frac{1}{2}}^{*}=\frac{\vec{w}_{j}^{n}+\overleftarrow{w}_{j+1}^{n}}{2}
$$

Notice that the source terms clearly appear to receive an upwind treatment in (31). This scheme is easily shown to be stable under the CFL condition given by

$$
\max _{j \in \mathbb{Z}} \frac{\Delta t}{\Delta m_{j}} a \leq \frac{1}{2},
$$

for all $j$. Let us note that as a satisfies the subcharacteristic condition (9) this CFL condition naturally involves the sound speed $c$.

\section{Implicit in time Godunov-type scheme for the Lagrangian system}

We follow here a standard approach for deriving an implicit in time Godunov-type scheme from (31) by simply replacing the terms evaluated at time $t^{n}$ with the terms noted with the superscript ${ }^{\mathrm{Lag}}$. We get

$$
\left\{\begin{array}{l}
\vec{w}_{j}^{\mathrm{Lag}}=\vec{w}_{j}^{n}-a \frac{\Delta t}{\Delta m_{j}}\left(\vec{w}_{j}^{\mathrm{Lag}}-\vec{w}_{j-1}^{\mathrm{Lag}}\right)+\Delta t a \frac{\Delta m_{j-1 / 2}}{\Delta m_{j}}\left(g-\alpha u_{j-\frac{1}{2}}^{*}\right) \\
\overleftarrow{w}_{j}^{\mathrm{Lag}}=\overleftarrow{w}_{j}^{n}+a \frac{\Delta t}{\Delta m_{j}}\left(\overleftarrow{w}_{j+1}^{\mathrm{Lag}}-\overleftarrow{w}_{j}^{\mathrm{Lag}}\right)-\Delta t a \frac{\Delta m_{j+1 / 2}}{\Delta m_{j}}\left(g-\alpha u_{j+\frac{1}{2}}^{*}\right)
\end{array}\right.
$$

with

$$
u_{j+\frac{1}{2}}^{*}=\frac{1}{2 a+\alpha \Delta m_{j+1 / 2}}\left(\vec{w}_{j}^{\mathrm{Lag}}-\overleftarrow{w}_{j+1}^{\mathrm{Lag}}+g \Delta m_{j+1 / 2}\right)
$$

and

$$
\left\{\begin{aligned}
\tau_{j}^{\mathrm{Lag}} & =\tau_{j}^{n}+\frac{\Delta t}{\Delta m_{j}}\left(u_{j+\frac{1}{2}}^{*}-u_{j-\frac{1}{2}}^{*}\right), \\
E_{j}^{\mathrm{Lag}} & =E_{j}^{n}-\frac{\Delta t}{\Delta m_{j}}\left((u p)_{j+\frac{1}{2}}^{*}-(u p)_{j-\frac{1}{2}}^{*}\right)+g \frac{\Delta t}{\Delta m_{j}} \frac{\Delta m_{j+1 / 2} u_{j+\frac{1}{2}}^{*}+\Delta m_{j-1 / 2} u_{j-\frac{1}{2}}^{*}}{2} \\
& -\alpha \frac{\Delta t}{\Delta m_{j}} \frac{\Delta m_{j+1 / 2}\left(u_{j+\frac{1}{2}}^{*}\right)^{2}+\Delta m_{j-1 / 2}\left(u_{j-\frac{1}{2}}^{*}\right)^{2}}{2},
\end{aligned}\right.
$$

with

$$
p_{j+\frac{1}{2}}^{*}=\frac{\vec{w}_{j}^{\mathrm{Lag}}+\overleftarrow{w}_{j+1}^{\mathrm{Lag}}}{2}
$$

It is important to notice that (34) is independent from (36). More precisely, once (34) is solved, the update values (36) for $\tau$ and $E$ follow explicitly. As far as $\vec{w}$ and $\overleftarrow{w}$ are concerned, the update formulas (34) are coupled and require the resolution of a linear system. The corresponding matrix is shown to be pentadiagonal and strictly diagonally dominant. Therefore, it is invertible and (34) can be solved for any $\Delta t>0$. The proposed implicit in time numerical scheme for solving the Lagrangian system is then actually cheap thanks to the relaxation strategy. 


\section{Implicit-explicit in time Godunov-type scheme for the Eulerian system (1)}

In order to complete the definition of our numerical scheme, it remains to define the second step of the operator splitting associated with the Lagrange-Projection decomposition, which corresponds to the Eulerian projection (5). Following [19], we consider a very classic upwind and explicit in time numerical scheme given by

$$
X_{j}^{n+1}=X_{j}^{\mathrm{Lag}}+\frac{\Delta t}{\Delta x}\left(\left(u_{j-\frac{1}{2}}^{*}\right)^{+} X_{j-1}^{\mathrm{Lag}}+\left[\left(u_{j+\frac{1}{2}}^{*}\right)^{-}-\left(u_{j-\frac{1}{2}}^{*}\right)^{+}\right] X_{j}^{\mathrm{Lag}}-\left(u_{j+\frac{1}{2}}^{*}\right)^{-} X_{j+1}^{\mathrm{Lag}}\right),
$$

where $X \in\{\rho, \rho u, \rho E\}$ and

$$
u^{+}=\frac{u+|u|}{2}, \quad u^{-}=\frac{u-|u|}{2},
$$

for all $u$. The update formula $(38)$ is shown to be stable under the CFL condition given by

$$
\frac{\Delta t}{\Delta x}\left(\left(u_{j-\frac{1}{2}}^{*}\right)^{+}-\left(u_{j+\frac{1}{2}}^{*}\right)^{-}\right)<1,
$$

see again [19] for instance. Note that this CFL condition involves only the flow speed and is not based on the acoustic waves.

For the sake of clarity, let us briefly recall the different steps of the overall method called LP-IMEX, suppose that at the instant $n$ we know $\left(\rho_{j}^{n},(\rho u)_{j}^{n},(\rho E)_{j}^{n}\right)$ for $j \in \mathbb{Z}$, we perform the following steps:

(i) compute $\left(\tau_{j}^{n}, \vec{w}_{j}^{n}, \overleftarrow{w}_{j}^{n}, E_{j}^{n}\right)$ at equilibrium by evaluating $\Pi_{j}^{n}=p\left(\rho_{j}^{n}, e_{j}^{n}\right)$

(ii) compute $\left(\tau_{j}^{\mathrm{Lag}}, \vec{w}_{j}^{\mathrm{Lag}}, \overleftarrow{w}_{j}^{\mathrm{Lag}}, E_{j}^{\mathrm{Lag}}\right)$ thanks to the implicit scheme defined by (34)-36,

(iii) evaluate $\left(\rho_{j}^{\mathrm{Lag}},(\rho u)_{j}^{\mathrm{Lag}},(\rho E)_{j}^{\mathrm{Lag}}\right)$ thanks to $\left(\tau_{j}^{\mathrm{Lag}}, \vec{w}_{j}^{\mathrm{Lag}}, \overleftarrow{w}_{j}^{\mathrm{Lag}}, E_{j}^{\mathrm{Lag}}\right)$

(iv) compute $\left(\rho_{j}^{n+1},(\rho u)_{j}^{n+1},(\rho E)_{j}^{n+1}\right)$ thanks to the explicit scheme defined by $(38)$ and 35.

In the numerical experiments and for the sake of comparison, we will also consider the following explicitexplicit numerical scheme that will be referred to as the LP-EXEX scheme. Suppose that at the instant $n$ we know $\left(\rho_{j}^{n},(\rho u)_{j}^{n},(\rho E)_{j}^{n}\right)$ for $j \in \mathbb{Z}$, we perform the following steps:

(i) compute $\left(\tau_{j}^{n}, \vec{w}_{j}^{n}, \overleftarrow{w}_{j}^{n}, E_{j}^{n}\right)$ at equilibrium by evaluating $\Pi_{j}^{n}=p\left(\rho_{j}^{n}, e_{j}^{n}\right)$

(ii) compute $\left(\tau_{j}^{\mathrm{Lag}}, \vec{w}_{j}^{\mathrm{Lag}}, \overleftarrow{w}_{j}^{\mathrm{Lag}}, E_{j}^{\mathrm{Lag}}\right)$ thanks to the explicit scheme defined by 31 ,

(iii) evaluate $\left(\rho_{j}^{\mathrm{Lag}},(\rho u)_{j}^{\mathrm{Lag}},(\rho E)_{j}^{\mathrm{Lag}}\right)$ thanks to $\left(\tau_{j}^{\mathrm{Lag}}, \vec{w}_{j}^{\mathrm{Lag}}, \overleftarrow{w}_{j}^{\mathrm{Lag}}, E_{j}^{\mathrm{Lag}}\right)$

(iv) compute $\left(\rho_{j}^{n+1},(\rho u)_{j}^{n+1},(\rho E)_{j}^{n+1}\right)$ thanks to the explicit scheme defined by 38 and 32 .

\section{Main properties}

We give in this section the main properties of the proposed numerical scheme.

Theorem 1. Under the CFL condition (39), the implicit-explicit in time numerical scheme LP-IMEX is well defined and satisfies the following stability properties:

(i) it is a conservative scheme for the density $\rho$. It is also a conservative scheme for $\rho u$ and $\rho E$ when the source terms are omitted,

(ii) the density $\rho_{j}^{n}$ is positive for all $j$ and $n>0$ provided that $\rho_{j}^{0}$ is positive for all $j$,

(iii) it is asymptotic preserving.

In addition, under the CFL condition (33), the explicit-explicit overall numerical scheme LP-EXEX

(iv) satisfies an entropy inequality.

This result is worth a few comments. We first note that the overall numerical scheme is stable under the CFL condition (39) which only involves the flow speed and not the acoustic waves. It is then less restrictive than the classical CFL restrictions of the usual explicit Godunov-type numerical schemes. The CFL condition (39) involves however the solution computed at the end of the Lagrangian step and then is not determined explicitly. Finding an explicit formula for the time step restriction that guarantees $(39)$ and then the stability of the scheme is an open question at the moment.

Properties (i) and (ii) are obtained from standard manipulations, see [19] and [14]. We first prove (iii) and then (iv). 


\subsection{Proof of (iii)}

This section aims at proving that the proposed LP-IMEX numerical scheme is asymptotic preserving. More precisely and similarly to the continuous analysis proposed in section 2 , we aim at proving that if we perform the scale change

$$
\Delta t \rightarrow \frac{\Delta t}{\epsilon}, \quad \alpha \rightarrow \frac{\alpha}{\epsilon}
$$

which corresponds to the study of the large time behaviour for a large friction coefficient, then we get a consistent approximation of (4) when $\epsilon$ goes to zero. We propose a two-part proof: we first prove the property in Lagrangian coordinates and then consider the Eulerian framework.

\subsubsection{Asymptotic preserving property in Lagrangian coordinates}

In Lagrangian coordinates, the limit system (4) reads

$$
\left\{\begin{array}{l}
\partial_{t} \tau-\partial_{m} u^{1}=0 \\
\partial_{m} p=g-\alpha u^{1} \\
\partial_{t} e+\partial_{m} p u^{1}=u^{1}\left(g-\alpha u^{1}\right)
\end{array}\right.
$$

Let us perform the scale change $(40)$ in $(34)-(36)$. We get

$$
\left\{\begin{aligned}
\vec{w}_{j}^{\mathrm{Lag}} & =\vec{w}_{j}^{n}-a \frac{\Delta t}{\epsilon \Delta m_{j}}\left(\vec{w}_{j}^{\mathrm{Lag}}-\vec{w}_{j-1}^{\mathrm{Lag}}\right)+\frac{\Delta t a}{\epsilon} \frac{\Delta m_{j-1 / 2}}{\Delta m_{j}}\left(g-\frac{\alpha}{\epsilon} u_{j-\frac{1}{2}}^{*}\right), \\
\overleftarrow{w}_{j}^{\mathrm{Lag}} & =\overleftarrow{w}_{j}^{n}+a \frac{\Delta t}{\epsilon \Delta m_{j}}\left(\overleftarrow{w}_{j+1}^{\mathrm{Lag}}-\overleftarrow{w}_{j}^{\mathrm{Lag}}\right)-\frac{\Delta t a}{\epsilon} \frac{\Delta m_{j+1 / 2}}{\Delta m_{j}}\left(g-\frac{\alpha}{\epsilon} u_{j+\frac{1}{2}}^{*}\right), \\
\tau_{j}^{\mathrm{Lag}} & =\tau_{j}^{n}+\frac{\Delta t}{\epsilon \Delta m_{j}}\left(u_{j+\frac{1}{2}}^{*}-u_{j-\frac{1}{2}}^{*}\right), \\
E_{j}^{\mathrm{Lag}} & =E_{j}^{n}-\frac{\Delta t}{\epsilon \Delta m_{j}}\left((u p)_{j+\frac{1}{2}}^{*}-(u p)_{j-\frac{1}{2}}^{*}\right)+\frac{g \Delta t}{\epsilon \Delta m_{j}} \frac{\Delta m_{j+1 / 2} u_{j+\frac{1}{2}}^{*}+\Delta m_{j-1 / 2} u_{j-\frac{1}{2}}^{*}}{2} \\
& -\frac{\alpha \Delta t}{\epsilon^{2} \Delta m_{j}} \frac{\Delta m_{j+1 / 2}\left(u_{j+\frac{1}{2}}^{*}\right)^{2}+\Delta m_{j-1 / 2}\left(u_{j-\frac{1}{2}}^{*}\right)^{2}}{2},
\end{aligned}\right.
$$

with

$$
u_{j+\frac{1}{2}}^{*}=\frac{\epsilon}{2 a \epsilon+\alpha \Delta m_{j+1 / 2}}\left(\vec{w}_{j}^{\mathrm{Lag}}-\overleftarrow{w}_{j+1}^{\mathrm{Lag}}+g \Delta m_{j+1 / 2}\right)
$$

and

$$
p_{j+\frac{1}{2}}^{*}=\frac{\vec{w}_{j}^{\mathrm{Lag}}+\overleftarrow{w}_{j+1}^{\mathrm{Lag}}}{2}
$$

Multiplying the first equation of 42 by $\epsilon$, we obtain $\vec{w}_{j}^{\text {Lag }}-\overleftarrow{w}_{j}^{\text {Lag }}=0+\mathcal{O}(\epsilon)$. This implies by definition of $\vec{w}$ and $\overleftarrow{w}$ that for $j \in \mathbb{Z}$

$$
u_{j}^{\mathrm{Lag}}=0+\mathcal{O}(\epsilon)
$$

which also gives

$$
p_{j+\frac{1}{2}}^{*}=P_{j+1 / 2}^{*}+\mathcal{O}(\epsilon)
$$

where

$$
P_{j+1 / 2}^{*}=\frac{\Pi_{j}^{\mathrm{Lag}}+\Pi_{j+1}^{\mathrm{Lag}}}{2}
$$

We also have thanks to 43 that

$$
u_{j+\frac{1}{2}}^{*}=\frac{\epsilon}{2 a \epsilon+\alpha \Delta m_{j+1 / 2}}\left(\vec{w}_{j}^{\mathrm{Lag}}-\overleftarrow{w}_{j+1}^{\mathrm{Lag}}+g \Delta m_{j+1 / 2}\right)=\epsilon v_{j+\frac{1}{2}}^{*}+\mathcal{O}\left(\varepsilon^{2}\right)
$$

where

$$
v_{j+\frac{1}{2}}^{*}=\frac{1}{\alpha}\left(g-\frac{\Pi_{j+1}^{\mathrm{Lag}}-\Pi_{j}^{\mathrm{Lag}}}{\Delta m_{j+1 / 2}}\right),
$$


and thus provide a definition of $v_{j+\frac{1}{2}}^{*}$ that is consistent with the second equation in 41 . By reinjecting the expression of $u_{j+\frac{1}{2}}^{*}$ in the last two equations of 42 , we obtain finally

$$
\left\{\begin{aligned}
\tau_{j}^{\mathrm{Lag}} & =\tau_{j}^{n}+\frac{\Delta t}{\Delta m_{j}}\left(v_{j+\frac{1}{2}}^{*}-v_{j-\frac{1}{2}}^{*}\right)+\mathcal{O}(\epsilon), \\
v_{j+\frac{1}{2}}^{*} & =\frac{1}{\alpha}\left(g-\frac{\Pi_{j+1}^{\mathrm{Lag}}-\Pi_{j}^{\mathrm{Lag}}}{\Delta m_{j}}\right), \\
P_{j+\frac{1}{2}}^{*} & =\frac{\Pi_{j}^{\mathrm{Lag}}+\Pi_{j+1}^{\mathrm{Lag}}}{2}, \\
e_{j}^{\mathrm{Lag}} & =e_{j}^{n}-\frac{\Delta t}{\Delta m_{j}}\left((v P)_{j+\frac{1}{2}}^{*}-(v P)_{j-\frac{1}{2}}^{*}\right)+g \frac{\Delta t}{\Delta m_{j}} \frac{\Delta m_{j+1 / 2} v_{j+\frac{1}{2}}^{*}+\Delta m_{j-1 / 2} v_{j-\frac{1}{2}}^{*}}{2} \\
& -\alpha \frac{\Delta t}{\Delta m_{j}} \frac{\Delta m_{j+1 / 2}\left(v_{j+\frac{1}{2}}^{*}\right)^{2}+\Delta m_{j-1 / 2}\left(v_{j-\frac{1}{2}}^{*}\right)^{2}}{2}+\mathcal{O}(\epsilon),
\end{aligned}\right.
$$

which is consistent with 41 when $\epsilon$ tends to 0 .

\subsubsection{Asymptotic Preserving property in Eulerian coordinates}

It remains to prove that after the Eulerian projection, the overall scheme is consistent with (4). In (38) we perform the scale change 40 . We have for $X \in\{\rho, \rho u, \rho E\}$ :

$$
X_{j}^{n+1}=X_{j}^{\mathrm{Lag}}+\frac{\Delta t}{\Delta x}\left(\left(v_{j-\frac{1}{2}}^{*}\right)^{+} X_{j-1}^{\mathrm{Lag}}+\left(\left(v_{j+\frac{1}{2}}^{*}\right)^{-}-\left(v_{j-\frac{1}{2}}^{*}\right)^{+}\right) X_{j}^{\mathrm{Lag}}-\left(v_{j+\frac{1}{2}}^{*}\right)^{-} X_{j+1}^{\mathrm{Lag}}\right)+\mathcal{O}(\epsilon) .
$$

Since we have $u_{j}^{\mathrm{Lag}}=0+\mathcal{O}(\epsilon)$, then $(\rho u)_{j}^{\mathrm{Lag}}=0+\mathcal{O}(\epsilon)$ for all $j \in \mathbb{Z}$ and considering $X=\rho u$ in the previous equality gives

$$
u_{j}^{n+1}=0+\mathcal{O}(\epsilon) .
$$

Let us remark that the first equation in 45 reads

$$
\rho_{j}^{n}=\rho_{j}^{\mathrm{Lag}}\left(1+\frac{\Delta t}{\Delta x}\left(v_{j+\frac{1}{2}}^{*}-v_{j-\frac{1}{2}}^{*}\right)\right)+\mathcal{O}(\epsilon) .
$$

Then, for $X \in\{\rho, \rho E\}$ the Eulerian projection 46 may be recast into

$$
(X)_{j}^{n+1}=(X)_{j}^{\mathrm{Lag}}\left(1+\frac{\Delta t}{\Delta x}\left(v_{j+\frac{1}{2}}^{*}-v_{j-\frac{1}{2}}^{*}\right)\right)-\mathscr{L}^{a}\left((X)^{\mathrm{Lag}}, v^{*}\right)+\mathcal{O}(\epsilon)=\rho_{j}^{n} \frac{X_{j}^{\mathrm{Lag}}}{\rho_{j}^{\mathrm{Lag}}}-\mathscr{L}^{a}\left((X)^{\mathrm{Lag}}, v^{*}\right)+\mathcal{O}(\epsilon),
$$

where the advection operator $\mathscr{L}^{a}\left((X)^{\mathrm{Lag}}, v^{*}\right)$ is consistent with $\partial_{x}(X v)$ and is defined by

$$
\mathscr{L}^{a}\left((X)^{\mathrm{Lag}}, v^{*}\right)=\frac{\Delta t}{\Delta x}\left\{\left[(X)_{j}^{\mathrm{Lag}}\left(v_{j+\frac{1}{2}}^{*}\right)^{+}+(X)_{j+1}^{\mathrm{Lag}}\left(v_{j+\frac{1}{2}}^{*}\right)^{-}\right]-\left[(X)_{j}^{\mathrm{Lag}}\left(v_{j-\frac{1}{2}}^{*}\right)^{-}+(X)_{j-1}^{\mathrm{Lag}}\left(v_{j-\frac{1}{2}}^{*}\right)^{+}\right]\right\} .
$$

Injecting the last equation of 45 into 477 yields in the limit $\epsilon \rightarrow 0$

$$
\left\{\begin{aligned}
\rho_{j}^{n+1} & =\rho_{j}^{n}-\mathscr{L}^{a}\left(\rho^{\mathrm{Lag}}, v^{*}\right)+\mathcal{O}(\epsilon), \\
v_{j+\frac{1}{2}}^{*} & =\frac{1}{\alpha}\left(g-2 \frac{\Pi_{j+1}^{\mathrm{Lag}}-\Pi_{j}^{\mathrm{Lag}}}{\left(\rho_{j}^{n}+\rho_{j+1}^{n}\right) \Delta x}\right), \\
P_{j+\frac{1}{2}}^{*} & =\frac{\Pi_{j}^{\mathrm{Lag}}+\Pi_{j+1}^{\mathrm{Lag}}}{2} \\
(\rho e)_{j}^{n+1} & =(\rho e)_{j}^{n}-\frac{\Delta t}{\Delta x}\left((v P)_{j+\frac{1}{2}}^{*}-(v P)_{j-\frac{1}{2}}^{*}\right) \\
& +\rho_{j}^{n} \Delta t\left(g \frac{v_{j+\frac{1}{2}}^{*}+v_{j-\frac{1}{2}}^{*}}{2}-\alpha \frac{\left(v_{j+\frac{1}{2}}^{*}\right)^{2}+\left(v_{j-\frac{1}{2}}^{*}\right)^{2}}{2}\right)-\mathscr{L}^{a}\left((\rho e)^{\mathrm{Lag}}, v^{*}\right)+\mathcal{O}(\epsilon),
\end{aligned}\right.
$$

which is clearly consistent with (4). Hence, the overall scheme is asymptotic preserving is the proof is completed. 


\subsection{Proof of (iv)}

We now prove that the explicit scheme LP-EXEX satisfies a discrete entropy inequality. As above, we first prove such an inequality in Lagrangian coordinates considering the scheme (31) that may be recast into

$$
\left\{\begin{aligned}
\tau_{j}^{\mathrm{Lag}} & =\tau_{j}^{n}+\frac{\Delta t}{\Delta m_{j}}\left(u_{j+\frac{1}{2}}^{*}-u_{j-\frac{1}{2}}^{*}\right), \\
u_{j}^{\mathrm{Lag}}= & u_{j}^{n}-\frac{\Delta t}{\Delta m_{j}}\left(p_{j+\frac{1}{2}}^{*}-p_{j-\frac{1}{2}}^{*}\right)+\frac{\Delta t}{\Delta m_{j}} \Delta m_{j-1 / 2}\left(g-\alpha u_{j-\frac{1}{2}}\right)+\frac{\Delta t}{\Delta m_{j}} \Delta m_{j+1 / 2}\left(g-\alpha u_{j+\frac{1}{2}}\right), \\
E_{j}^{\mathrm{Lag}}=E_{j}^{n}-\frac{\Delta t}{\Delta m_{j}}\left((u p)_{j+\frac{1}{2}}^{*}-(u p)_{j-\frac{1}{2}}^{*}\right)+g \frac{\Delta t}{\Delta m_{j}} \frac{\Delta m_{j+1 / 2} u_{j+\frac{1}{2}}^{*}+\Delta m_{j-1 / 2} u_{j-\frac{1}{2}}^{*}}{2} & \\
& -\alpha \frac{\Delta t}{\Delta m_{j}} \frac{\Delta m_{j+1 / 2}\left(u_{j+\frac{1}{2}}^{*}\right)^{2}+\Delta m_{j-1 / 2}\left(u_{j-\frac{1}{2}}^{*}\right)^{2}}{2},
\end{aligned}\right.
$$

with

$$
u_{j+\frac{1}{2}}^{*}=\frac{1}{2 a+\alpha \Delta m_{j+1 / 2}}\left(a\left(u_{j+1}^{n}+u_{j}^{n}\right)-\left(p\left(\rho_{j+1}^{n}, e_{j+1}^{n}\right)-p\left(\rho_{j}^{n}, e_{j}^{n}\right)\right)+g \Delta m_{j+1 / 2}\right)
$$

and

$$
p_{j+\frac{1}{2}}^{*}=\frac{p\left(\rho_{j+1}^{n}, e_{j+1}^{n}\right)+p\left(\rho_{j}^{n}, e_{j}^{n}\right)}{2}-\frac{a\left(u_{j+1}^{n}-u_{j}^{n}\right)}{2} .
$$

Then we take the Eulerian projection (38) into account to obtain a discrete entropy inequality for the overall scheme in Eulerian coordinates.

Let $s=s(\rho, e)$ be the strictly convex mathematical entropy. The entropy inequality associated with (1) writes

$$
\partial_{t}(\rho s)+\partial_{x}(\rho u s) \leq 0
$$

In Lagrangian coordinates, (7) is associated with the following entropy inequality

$$
\partial_{t} s \leq 0 .
$$

In the sequel and with a little abuse in the notations, we will consider the pressure as a function of the density and the entropy $p=p(\rho, s)$.

\subsubsection{Entropy inequality in Lagrangian coordinates}

The scheme (48) is a Godunov-type scheme. We prove here that the associated approximate Riemann solver is consistent with the entropy inequality (50). Let us check that

$$
0 \leq-a\left(s_{L}^{*}-s_{L}\right)+a\left(s_{R}-s_{R}^{*}\right),
$$

where $s_{L}^{*}=s\left(\rho_{L}^{*}, e_{L}^{*}\right)$ and $s_{R}^{*}=s\left(\rho_{R}^{*}, e_{R}^{*}\right)$ so that 21) is verified with $\eta=s, q=0$ and $\tilde{\sigma}\left(\Delta m, \Delta t ; \mathbf{U}_{L}, \mathbf{U}_{R}\right)=0$. Let us first prove the following result.

Proposition 1. If $a>0$ is such that

$$
\left\{\begin{array}{l}
\rho_{L}^{*}>0, \quad \rho_{R}^{*}>0, \\
\rho^{2} \partial_{\rho} p\left(\rho, s_{L}\right) \leq a^{2}, \quad \forall \rho \in I\left(\rho_{L}, \rho_{L}^{*}\right), \\
\rho^{2} \partial_{\rho} p\left(\rho, s_{R}\right) \leq a^{2}, \quad \forall \rho \in I\left(\rho_{R}, \rho_{R}^{*}\right)
\end{array}\right.
$$

then we have

$$
\left\{\begin{array}{l}
e_{L}^{*} \geq e\left(\rho_{L}^{*}, s_{L}\right) \\
e_{R}^{*} \geq e\left(\rho_{R}^{*}, s_{R}\right)
\end{array}\right.
$$

Proof. Recall that we have

$$
\left\{\begin{array}{l}
E_{L}^{*}=E_{L}+\frac{1}{a}\left(p_{L} u_{L}-u^{*}\left[p^{*}-\frac{\Delta m}{2}\left(g-\alpha u^{*}\right)\right]\right), \\
E_{R}^{*}=E_{R}+\frac{1}{a}\left(u^{*}\left[p^{*}+\frac{\Delta m}{2}\left(g-\alpha u^{*}\right)\right]-p_{R} u_{R}\right),
\end{array}\right.
$$


and

$$
\left\{\begin{array}{l}
\Pi_{R}^{*}=p^{*}+\frac{\Delta m}{2}\left(g-\alpha u^{*}\right) \\
\Pi_{L}^{*}=p^{*}-\frac{\Delta m}{2}\left(g-\alpha u^{*}\right)
\end{array}\right.
$$

which gives

$$
\left\{\begin{array}{l}
E_{L}^{*}=E_{L}-\frac{1}{a}\left(\Pi_{L}^{*} u^{*}-\Pi_{L} u_{L}\right) \\
E_{R}^{*}=E_{R}+\frac{1}{a}\left(\Pi_{R}^{*} u^{*}-\Pi_{R} u_{R}\right)
\end{array}\right.
$$

so that

$$
e_{R}^{*}=E_{R}^{*}-\frac{1}{2} u^{* 2}=e_{R}+\frac{1}{2}\left(u_{R}^{2}-u^{* 2}\right)+\frac{1}{a}\left(\Pi_{R}^{*} u^{*}-\Pi_{R} u_{R}\right)
$$

Using the definitions of $u^{*}$ and $\Pi_{R}^{*}$ given in $(30)$, straightforward calculations then lead to

$$
e_{R}^{*}=e_{R}+\frac{1}{2 a^{2}}\left(\Pi_{R}^{* 2}-\Pi_{R}^{2}\right)
$$

and

$$
\Pi_{R}^{*}=\Pi_{R}+a\left(u^{*}-u_{R}\right) .
$$

The latter equality, together with the last two equalities in (27), gives

$$
\frac{\Pi_{R}^{*}}{a^{2}}=\frac{1}{\rho_{R}}+\frac{p_{R}}{a^{2}}-\frac{1}{\rho_{R}^{*}} .
$$

It is then not difficult to check that

$$
\left\{\begin{aligned}
e_{R}^{*}-e\left(\rho_{R}^{*}, s_{R}\right) & =\frac{1}{2 a^{2}}\left(p\left(\rho_{R}^{*}, s_{R}\right)-\Pi_{R}^{*}\right)^{2}+\phi\left(\rho_{R}\right), \\
\phi(\rho) & =e\left(\rho, s_{R}\right)-\frac{p\left(\rho, s_{R}\right)^{2}}{2 a^{2}}-e\left(\rho_{R}^{*}, s_{R}\right)+\frac{p\left(\rho_{R}^{*}, s_{R}\right)^{2}}{2 a^{2}}+p\left(\rho_{R}^{*}, s_{R}\right)\left(\frac{1}{\rho}+\frac{p\left(\rho, s_{R}\right)}{a^{2}}-\frac{1}{\rho_{R}^{*}}-\frac{p\left(\rho_{R}^{*}, s_{R}\right)}{a^{2}}\right)
\end{aligned}\right.
$$

with, using the well-known relation (2),

$$
\left\{\begin{array}{l}
\phi^{\prime}(\rho)=\left(p\left(\rho, s_{R}\right)-p\left(\rho_{R}^{*}, s_{R}\right)\right)\left(\frac{1}{\rho^{2}}-\frac{1}{a^{2}} \partial_{\rho} p\left(\rho, s_{R}\right)\right), \\
\phi\left(\rho_{R}^{*}\right)=0 .
\end{array}\right.
$$

Therefore $\phi(\rho) \geq 0$ for all $\rho \in I\left(\rho_{R}, \rho_{R}^{*}\right)$ since $\partial_{\rho} p \geq 0$ under the assumptions of Weyl and by (9). We have thus proved in particular that

$$
e_{R}^{*} \geq e\left(\rho_{R}^{*}, s_{R}\right) .
$$

The proof of the second inequality follows the same idea and the proof of the proposition is then completed.

In order to get (51), we then note that the temperature $T$ being positive by assumption, we have $\partial_{e} s(\rho, e)<0$ where $s=s(\rho, e(\rho, s))$. So that

$$
s_{R}-s_{R}^{*}=s\left(\rho_{R}^{*}, e\left(\rho_{R}^{*}, s_{R}\right)\right)-s\left(\rho_{R}^{*}, e_{R}^{*}\right)=\partial_{e} s\left(\rho_{R}^{*}, \bar{e}_{L}^{*}\right)\left(e\left(\rho_{R}^{*}, s_{R}\right)-e_{R}^{*}\right) \geq 0,
$$

that is

$$
s_{R}-s_{R}^{*} \geq 0
$$

We get in the same way $s_{L}-s_{L}^{*} \geq 0$ which gives (51) since $a>0$. Then, under the CFL condition (33), the scheme in Lagrangian coordinates satisfies the discrete entropy inequality 23 which reads

$$
s_{j}^{\mathrm{Lag}} \leq s_{j}^{n}-\frac{\Delta t}{\Delta m_{j}}\left(q_{j+\frac{1}{2}}^{n}-q_{j-\frac{1}{2}}^{n}\right)
$$

where $q_{j+\frac{1}{2}}^{n}$ naturally follows from 24 . 


\subsubsection{Entropy inequality in Eulerian coordinates}

Let us now prove an entropy inequality for the whole Lagrange-Projection scheme. We first recall that the function $\rho s=\rho s(\rho, \rho u, \rho E)$ is strictly convex by assumption, see [19]. The Eulerian projection (38) being a convex combination for each $X=\rho, \rho u, \rho E$, we thus have

$$
(\rho s)_{j}^{n+1} \leq(\rho s)_{j}^{\mathrm{Lag}}+\frac{\Delta t}{\Delta x}\left(\left(u_{j-\frac{1}{2}}^{*}\right)^{+}(\rho s)_{j-1}^{\mathrm{Lag}}+\left(\left(u_{j+\frac{1}{2}}^{*}\right)^{-}-\left(u_{j-\frac{1}{2}}^{*}\right)^{+}\right)(\rho s)_{j}^{\mathrm{Lag}}-\left(u_{j+\frac{1}{2}}^{*}\right)^{-}(\rho s)_{j+1}^{\mathrm{Lag}}\right) .
$$

Then using the first equation of (48) in (54), together with the definition of $\Delta m_{j}$, we have

$$
(\rho s)_{j}^{\mathrm{Lag}} \leq(\rho s)_{j}^{n}-\frac{\Delta t}{\Delta x}\left(q_{j+\frac{1}{2}}^{n}-q_{j-\frac{1}{2}}^{n}\right)-\frac{\Delta t}{\Delta x}(\rho s)_{j}^{\mathrm{Lag}}\left(u_{j+\frac{1}{2}}^{*}-u_{j-\frac{1}{2}}^{*}\right) .
$$

Then using these two inequalities together with the simple relation $u=u^{+}+u^{-}$, we get

$$
(\rho s)_{j}^{n+1} \leq(\rho s)_{j}^{n}-\frac{\Delta t}{\Delta x}\left(g_{j+\frac{1}{2}}^{n}-g_{j-\frac{1}{2}}^{n}\right),
$$

where

$$
g_{j+\frac{1}{2}}^{n}=\left(u_{j+\frac{1}{2}}^{*}\right)^{+}(\rho s)_{j}^{\mathrm{Lag}}+\left(u_{j+\frac{1}{2}}^{*}\right)^{-}(\rho s)_{j+1}^{\mathrm{Lag}}+q_{j+\frac{1}{2}}^{n} .
$$

Which is nothing but the expected discrete entropy inequality for the whole Lagrange-Projection scheme (note indeed that $g_{j+\frac{1}{2}}^{n}$ is clearly consistent with the entropy flux $\rho s u$ ).

\section{$9 \quad$ Numerical results}

For the sake of stressing the importance of the source term discretization, we propose to also consider in the following a more simple approximation strategy that will be referred to as the LP-EXEX SP scheme. In this scheme, the source terms will be treated by means of a separate operator splitting. Suppose that for some instant $n$ we know $\left(\rho_{j}^{n},(\rho u)_{j}^{n},(\rho E)_{j}^{n}\right)$ for $j \in \mathbb{Z}$, the LP-EXEX SP numerical scheme reads:

(i) compute $\left(\tau_{j}^{n}, \vec{w}_{j}^{n}, \overleftarrow{w}_{j}^{n}, E_{j}^{n}\right)$ at equilibrium by evaluating $\Pi_{j}^{n}=p\left(\rho_{j}^{n}, e_{j}^{n}\right)$

(ii) compute $\left(\tau_{j}^{\text {Lag }}, \vec{w}_{j}^{\text {Lag }}, \overleftarrow{w}_{j}^{\text {Lag }}, E_{j}^{\text {Lag }}\right)$ thanks to the use the explicit scheme defined by 31 with $\alpha=0$ and $g=0$, so that the gravity and friction terms are not taken into account yet

(iii) evaluate $\left(\rho_{j}^{\text {Lag }},(\rho u)_{j}^{\text {Lag }},(\rho E)_{j}^{\text {Lag }}\right)$ thanks to $\left(\tau_{j}^{\text {Lag }}, \vec{w}_{j}^{\text {Lag }}, \overleftarrow{w}_{j}^{\text {Lag }}, E_{j}^{\text {Lag }}\right)$

(iv) compute $\left(\rho_{j}^{n+1, \sharp},(\rho u)_{j}^{n+1, \sharp},(\rho E)_{j}^{n+1, \sharp}\right)$ thanks to the explicit scheme defined by 38 and 32 ,

(v) account for the gravity and friction source terms by integrating the system of ordinary differential equations

$$
\frac{\mathrm{d}}{\mathrm{d} t}\left[\begin{array}{c}
\rho \\
\rho u \\
\rho E
\end{array}\right]=\left[\begin{array}{c}
0 \\
\rho(g-\alpha u) \\
\rho u(g-\alpha u)
\end{array}\right]
$$

The update in term of the variable $\rho, u$, and $e$ then reads

$$
\left\{\begin{array}{l}
\rho_{j}^{n+1}=\rho_{j}^{n+1, \sharp}, \\
u_{j}^{n+1}=u_{j}^{n+1, \sharp} e^{-\alpha \Delta t}+\frac{g}{\alpha}\left(1-e^{-\alpha \Delta t}\right), \\
e_{j}^{n+1}=e_{j}^{n+1, \sharp} .
\end{array}\right.
$$

(vi) evaluate $\left(\rho_{j}^{n+1},(\rho u)_{j}^{n+1},(\rho E)_{j}^{n+1}\right)$.

We propose to test both LP-EXEX SP and LP-IMEX scheme against a test case that has been proposed in [11. In the sequel, we shall consider that the fluid is equipped with a perfect gas equation of state $p=(\gamma-1) \rho e$ and we set the gravity acceleration, the friction coefficient and the specific heat ratio to the following values

$$
g=9.81 \mathrm{~m} \cdot \mathrm{s}^{-2}, \quad \alpha=10^{6} \mathrm{~s}^{-1}, \quad \gamma=1.4 .
$$

The initial condition is defined by

$$
\begin{cases}(\rho, u, p)=(1.0,0,10000.0), & \text { if } x \in[0,0.35] \cap[0.65,1], \\ (\rho, u, p)=(2.0,0,26390.2), & \text { if } x \in[0.35,0.65] .\end{cases}
$$


Table 1: Comparison of the relative errors between the approximated solutions obtained with both LP-EXEX $\mathrm{SP}$ and LP-IMEX schemes. The space domain is discretized with a 1000 -cell space discretization and $\Delta t=\frac{1}{\alpha}$ for both schemes.

\begin{tabular}{rccc}
\hline numerical scheme & $\operatorname{err}(\rho, t=0.01)$ & $\operatorname{err}(u, t=0.01)$ & $\operatorname{err}(P, t=0.01)$ \\
\hline LP-EXEX SP & $1.686931 \times 10^{-2}$ & $6.858335 \times 10^{-1}$ & $2.539820 \times 10^{-2}$ \\
LP-IMEX & $3.959560 \times 10^{-4}$ & $1.195630 \times 10^{-2}$ & $5.635518 \times 10^{-4}$ \\
\hline
\end{tabular}

At the boundaries, we impose periodic boundary conditions thanks to a fictious cell at each end of the domain. In the sequel, both LP-EXEX and LP-EXEX SP computations will be performed with a time step defined by

$$
\Delta t=\frac{\min \left(\rho_{j}^{n}\right) \Delta x}{2 a},
$$

in order to agree with the classic acoustic CFL (33). The choice of the time step for the LP-IMEX scheme will be specified case by case. For each test, we will compute a reference solution thanks to the LP-EXEX scheme over a 10000 -cell grid. If we refer to this solution thanks to the superscript ${ }^{\text {ref }}$ and if $Y$ denote a fluid variable, for the sake of comparison we shall consider in the sequel the $L^{1}$ relative error with respect to the reference solution at the instant $t$ defined by

$$
\operatorname{err}(Y, t)=\frac{\left\|Y(\cdot, t)-Y^{\mathrm{ref}}(\cdot, t)\right\|_{L^{1}([0,1])}}{\left\|Y^{\mathrm{ref}}(\cdot, t)\right\|_{L^{1}([0,1])}}
$$

\subsection{Test case 1: sensitivity with respect to the space step for large friction}

We run our numerical tests with the LP-EXEX SP scheme using a spatial discretization over 100 cells, 1000 cells and 10000 cells. This leads to time step values $\Delta t$ that are respectively of magnitude $\frac{10}{\alpha}, \frac{1}{\alpha}$ and $\frac{1}{10 \alpha}$. In figure 3. we display the result obtained at $t=0.01 \mathrm{~s}$ and we can see that there is a large amount of numerical diffusion due to the discretization of the source term with large values of $\alpha$. It is necessary to choose small values of $\Delta t$ relatively to $\frac{1}{\alpha}$ in order to preserve the accuracy of the solution.

We now consider the same test performed with the LP-IMEX scheme. We now choose $\Delta t$ in agreement with the CFL condition (39) by setting

$$
\Delta t=\min \left(\frac{\Delta x}{2 \max \left(u_{j}^{n}\right)}, \frac{1}{\alpha}\right),
$$

so that we always have $\Delta t \leq \frac{1}{\alpha}$. The results obtained with LP-IMEX scheme at instant $t=0.01 \mathrm{~s}$ are presented in figure 4 for discretization grids of 100 cells and 1000 cells. It is clear that the approximate solution is much more accurate than the one computed with the LP-EXEX SP scheme, even for coarse mesh. Let us note that for the relatively large space steps that have been used here, choice 58 imposes $\Delta t=\frac{1}{\alpha}$. Table 1 displays the relative error obtained with both the LP-EXEX SP and the LP-IMEX scheme. It shows that the asymptotic preserving property significantly lessen the numerical diffusion and then improve accuracy by several orders of magnitude.

\subsection{Test case 2: sensitivity with respect to the time step}

We are now interested in testing the LP-IMEX scheme in situations where $\Delta t$ is much bigger than $\frac{1}{\alpha}$. In order to procede, we relax the previous time step choice (58) by suppressing the control of with respect to $\frac{1}{\alpha}$. We simply choose to define $\Delta t$ in agreement with the CFL condition (39) based on the material velocity by setting

$$
\Delta t=\min \left(\frac{\Delta x}{2 \max \left(u_{j}^{n}\right)}\right) .
$$



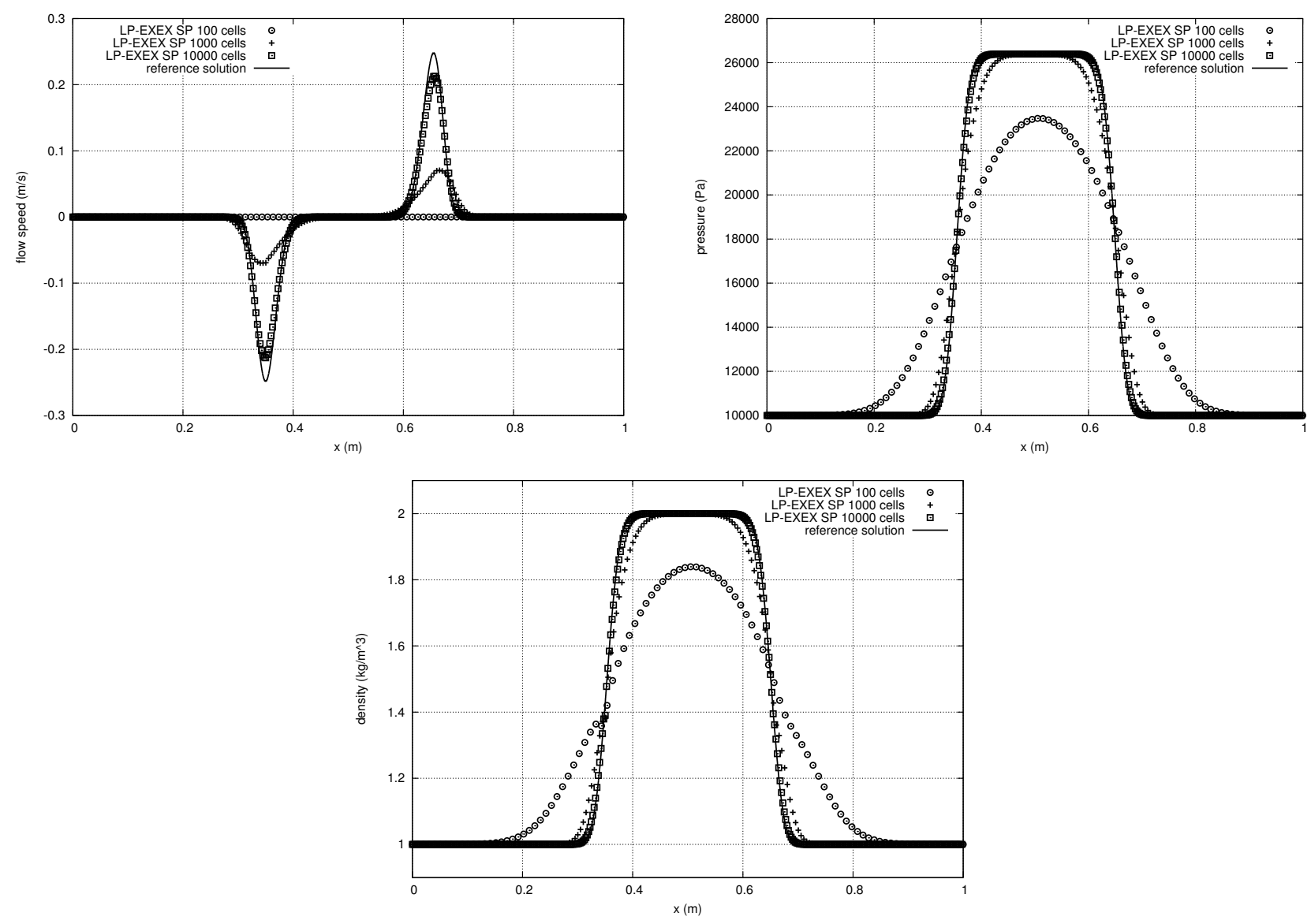

Figure 3: Profile at time $t=0.01 \mathrm{~s}$ of the velocity (top left), the pressure (top right), the density (bottom) obtained for a 100-cell, 1000-cell and 10000-cell grid with the LP-EXEX SP scheme and the reference solution (LP-EXEX scheme with 10 000-cell mesh).

The test is performed with a 1000-cell mesh. The graph of the approximate solution at $t=0.01 \mathrm{~s}$ are displayed in figure 5. For this grid choice, we obtain that the magnitude of $\Delta t$ is $\frac{1000}{\alpha}$. Let us underline that these time steps are 1000 times larger than the time steps used in section 9.1. It appears that even for such relatively large time step and space step the approximate solution remains very accurate. This results is the consequence of the good behaviour of the numerical scheme for large friction coefficients. We compare in table 2 the relative errors of the fluid variables for this choice of time step values. We can verify here that the LP-IMEX scheme enables the use of time steps that are much larger than the acoustic based time step and also much larger than $\frac{1}{\alpha}$ while preserving accurate simulation results.

\section{Conclusion}

We designed a large time step and asymptotic preserving scheme for the gas dynamics equations with gravity and linear friction. The stability is proved under a time step CFL restriction based on the velocity $u$ only and not on the sound speed $c$. The scheme gives very good results and combines accuracy thanks to the asymptotic preserving property and efficiency thanks to the large time step stability condition. Future developments include an extension to several space dimensions and high-order accuracy, to low-Mach number flows and to more complicated systems of partial differential equations used in the modeling of two phase flows.

Acknowledgement. This present work has been achieved within the framework of the research project LATSAP. This project was funded by the CEA/DEN/DANS/DM2S for the 2011 edition of the CEMRACS. 

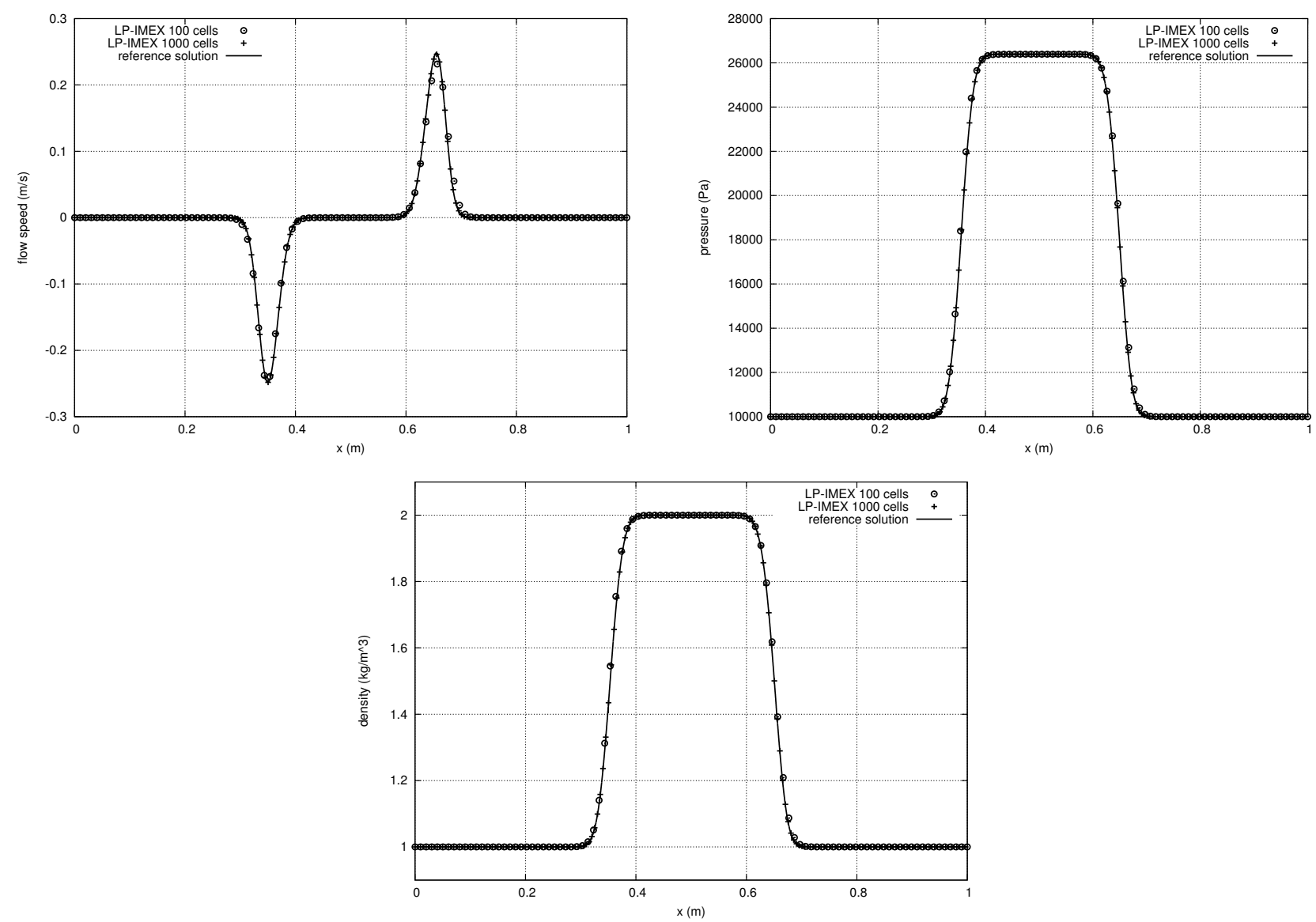

Figure 4: Profile at time $t=0.01 \mathrm{~s}$ of the velocity (top left), the pressure (top right), the density (bottom) obtained for a 100-cell and 1000-cell grid with the LP-IMEX scheme and the reference solution (LP-EXEX scheme with 10000 -cell mesh).

\section{References}

[1] R. Abgrall, D. Aregba, C. Berthon, M. Castro, C. Parés Special issue : Numerical approximations of hyperbolic systems with source terms and applications, J. Sci. Comput. 48 (2011)

[2] C. Berthon, P. Charrier and B. Dubroca, An HLLC scheme to solve the M1 Model of radiative transfer in two space dimensions, J. Sci. Comput. 31, no. 3, (2007) 347-389

[3] C. Berthon, P. G. LeFloch, R. Turpault, Late-time relaxation limits of nonlinear hyperbolic systems. A general framework, Math. of Comput., to appear

[4] C. Berthon and R. Turpault. Asymptotic preserving HLL schemes. Numer. Methods Partial Differential Equations, 27 (6) (2011) pp 1396-1422.

[5] F. Bouchut. A reduced stability condition for nonlinear relaxation to conservative laws. J. Hyp. Diff. Eq., 1(1), pp. 149-170, (2004).

[6] F. Bouchut, H. Ounaissa and B. Perthame, Upwinding of the source term at interfaces for Euler equations with high friction, Comput. Math. Appl. 53 (2007) no. 3-4, 361-375.

[7] C. Buet and S. Cordier. An asymptotic preserving scheme for hydrodynamics radiative transfer models: numerics for radiative transfer, Numer. Math. 108 (2007) no. 2, 199-221.

[8] C. Buet and B. Després, Asymptotic preserving and positive schemes for radiation hydrodynamics, J. Comput. Phys. 215 (2006) no. 2, 717-740. 
Table 2: Comparison of the relative $L^{1}$-errors obtained with the LP-IMEX scheme for a 1000 -cell space discretization and two different $\Delta t$ values.

\begin{tabular}{rcccc}
\hline numerical scheme & $\Delta t$ & $\operatorname{err}(\rho, t=0.01)$ & $\operatorname{err}(u, t=0.01)$ & $\operatorname{err}(P, t=0.01)$ \\
\hline LP-IMEX & $\frac{1}{\alpha}$ & $3.959560 \times 10^{-4}$ & $1.195630 \times 10^{-2}$ & $5.635518 \times 10^{-4}$ \\
LP-IMEX & $\frac{1000}{\alpha}$ & $2.607495 \times 10^{-3}$ & $1.099137 \times 10^{-1}$ & $3.288768 \times 10^{-3}$ \\
\hline
\end{tabular}
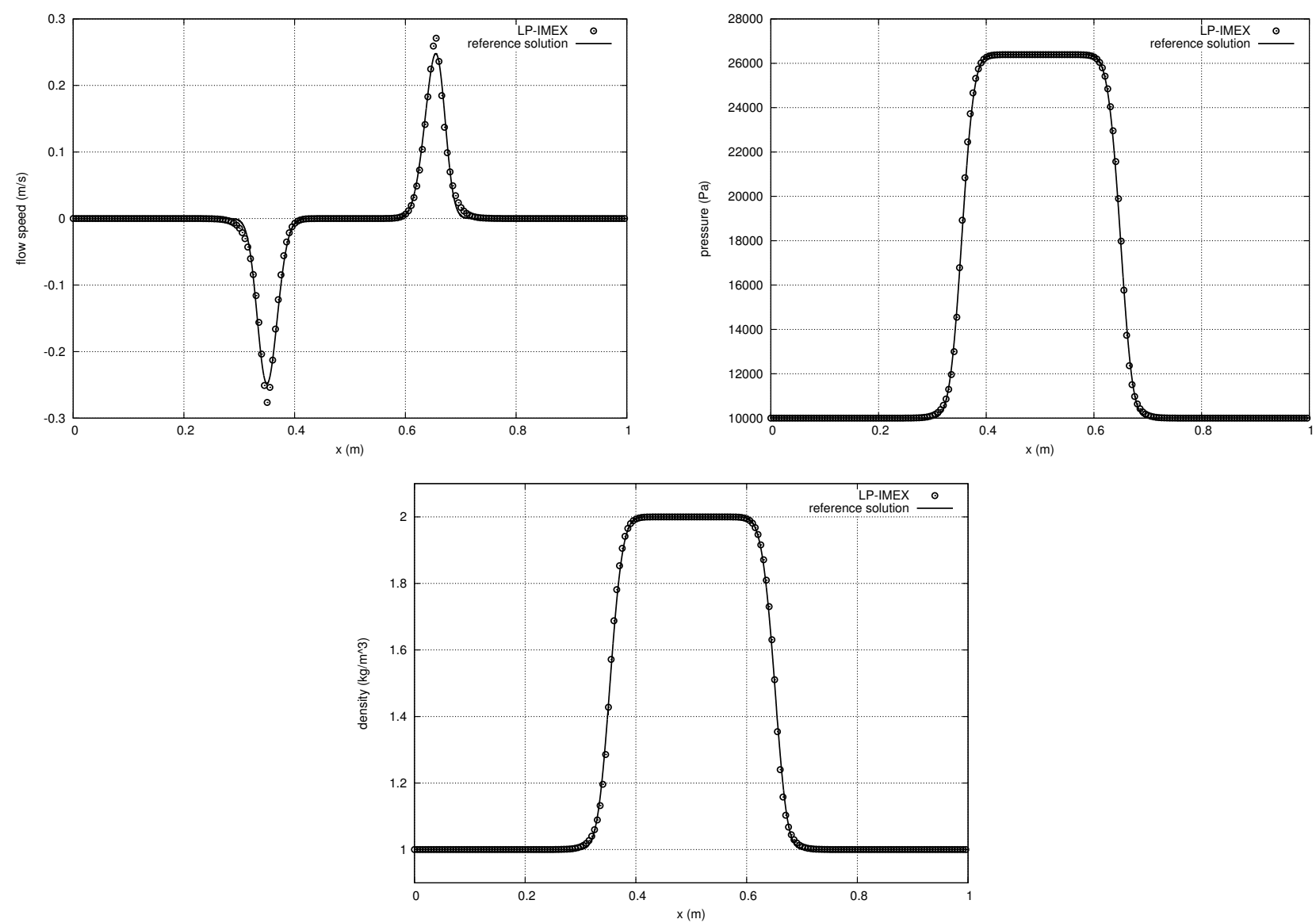

Figure 5: Profile at time $t=0.01 \mathrm{~s}$ of the velocity (top left), the pressure (top right), the density (bottom) obtained for a 1000-cell grid with the LP-IMEX scheme and the reference solution (LP-EXEX scheme with 10000 -cell mesh).

[9] R. Caflisch, R., S. Jin and G. Russo, Uniformly accurate schemes for hyperbolic systems with relaxation, SIAM J. Numer. Anal. 34 (1997) no. 1, 246-281.

[10] P. Cargo and A.-Y. Le Roux. Un schéma équilibre adapté au modèle d'atmosphère avec termes de gravité. C. R. Acad. Sci. Paris, Série I 318, pp. 73-76, (1994).

[11] C. Chalons, F. Coquel, E. Godlewski, P-A Raviart, N. Seguin. Godunov-type schemes for hyperbolic systems with parameter dependent source. The case of Euler system with friction. Math. Models Methods Appl. Sci., (2010).

[12] C. Chalons and J.F. Coulombel. Relaxation approximation of the Euler equations. Journal of Mathematical Analysis and Applications, 348(2), pp. 872-893, (2008). 
[13] F. Chalons, C. Coquel. Navier-stokes equations with several independant pressure laws and explicit predictor-corrector schemes. Numerisch Math, 101(3), pp. 451-478, (2005).

[14] F. Coquel, Q.L. Nguyen, M. Postel, and Q.H. Tran. Entropy-satisfying relaxation method with large timesteps for Euler IBVPs. Math. Comp. 79, pp. 1493-1533, (2010).

[15] F. Coquel, E. Godlewski, B. Perthame, A. In and P. Rascle. Some new Godunov and relaxation methods for two-phase flow problems, In Godunov methods (Oxford, 1999) (Kluwer/Plenum, 2001) pp. 179-188.

[16] M. Dumbser, C. Enaux C. and E.F. Toro, Finite volume schemes of very high order of accuracy for stiff hyperbolic balance laws, Journal of Computational Physics 227 (2008) 3971-4001.

[17] G. Gallice. Solveurs simples positifs et entropiques pour les systèmes hyperboliques avec terme source. C. R. Math. Acad. Sci. Paris 334, no. 8, pp. 713-716, (2002).

[18] G. Gallice. Positive and entropy stable Godunov-type schemes for gas dynamics and MHD equations in Lagrangian or Eulerian coordinates. Numer. Math. 94 no. 4, pp. 673-713, (2003).

[19] E. Godlewski and P.-A. Raviart. Numerical approximation of hyperbolic systems of conservation laws. Applied Mathematical Sciences, vol. 118, Springer-Verlag, New York, (1996).

[20] L. Gosse and G. Toscani, An asymptotic-preserving well-balanced schemes for the hyperbolic heat equations, C. R. Acad. Sci. Paris, Ser. I 334 (2002) 337-342.

[21] L. Gosse and G. Toscani, Space Localization and Well-Balanced Schemes for Discrete Kinetic Models in Diffusive Regimes, SIAM J. Numer. Anal. 41 (2003) no. 2, 641-658.

[22] L. Gosse and G. Toscani, Asymptotic-preserving and well-balanced schemes for radiative transfer and the Rosseland approximation, Numer. Math. 98 (2004) no. 2, 223-250.

[23] S. Jin. Runge-Kutta methods for hyperbolic conservation laws with stiff relaxation terms, J. Comput. Phys. 122 (1995) 51-67.

[24] S. Jin. Efficient asymptotic-preserving (AP) schemes for some multiscale kinetic equations, SIAM J. Sci. Comput. 21 (1999) 441-454.

[25] S. Jin and C.D. Levermore, Numerical Schemes for Hyperbolic Conservation Laws with Stiff Relaxation Terms, J. Computational Physics 126 (1996) 449-467.

[26] A. Klar, An asymptotic-induced scheme for nonstationary transport equations in the diffusive limit, SIAM J. Numer. Anal. 35 (1998) no. 3, 1073-1094.

[27] S. Jin and Z. P. Xin. The relaxation schemes for systems of conservation laws in arbitrary space dimension. Comm. Pure Appl. Math. 48, no. 3, pp. 235-276, (1995).

[28] T.-P. Liu. Hyperbolic conservation laws with relaxation. Comm. Math. Phys. 108, no. 1, pp. 153-175, (1987).

[29] G. Naldi and L. Pareschi, Numerical schemes for hyperbolic systems of conservation laws with stiff diffusive relaxation, SIAM J. Numer. Anal. 37 (2000) no. 4, 1246-1270.

[30] Roberto Natalini. Convergence to equilibrium for the relaxation approximations of conservation laws. Comm. Pure Appl. Math. 49, pp. 1-30, (1996).

[31] I. Suliciu. Ont the thermodynamics of fluids with relaxation and phase transitions. Fluids with relaxation. Int. J. Engag. Sci. 36, pp. 921-947, (1998).

[32] H. Weyl. Shock waves in arbitrary fluids. Comm. Pure Appl. Math 2, pp. 103-122, (1949). 